2. To: (Receiving Organization) Distribution

5. Proj./Prog./Dept./Div.:

Year 2000 TWRS Maintenance

Procedure Review

Report/Waste

Management/TBP/Process

Engineering

8. Originator Remarks:

This document is being released into the supporting document system for retrievability purposes.

11. Receiver Remarks: 11A. Design Baseline Document? [] Yes [X] No

For release.
3. From: (originating Organization)

Data Management/Technical

Basis and Planning

6. Design Authority/ Design Agent/Cog. Engr.:

M. R. Adams
4. Related EDT No.:

N/A

7. Purchase Order No.:

$N / A$

9. Equip./Component No.: $\mathrm{N} / \mathrm{A}$

10. System/Bldg./Facility: N/A

12. Major Assm. Dwg. No:: N/A

13. Permit/Permit Application No.: N/A

14. Required Response Date: $09 / 21 / 98$

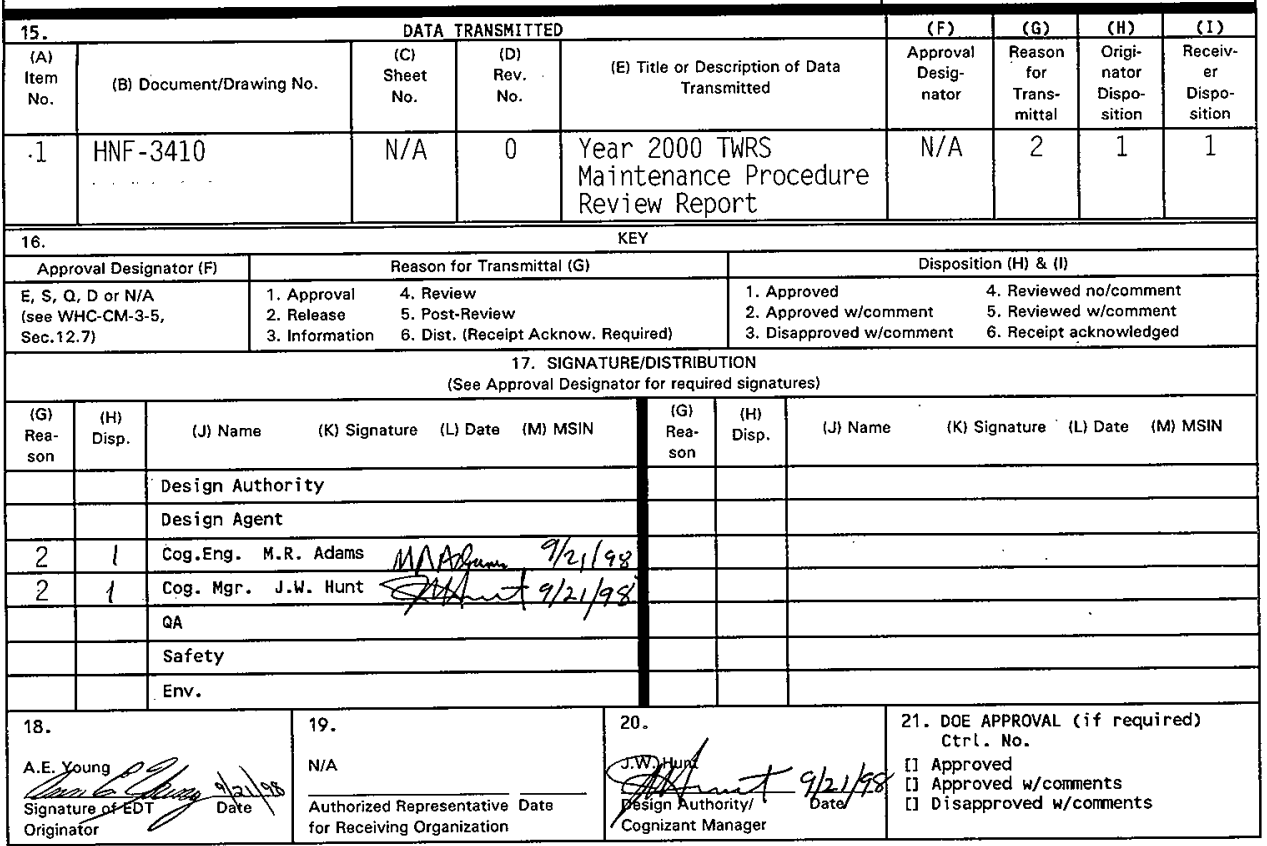




\section{Year 2000 TWRS Maintenance Procedure Review Report}

M. R. Adams

Lockheed Martin Hanford, Corp., Richland, WA 99352

U.S. Department of Energy Contract DE-AC06-87RL10930

EDT/ECN: EDT-622476 UC: 2070

Org Code: 7A110 Charge Code: D1Y11

B\&R Code: EW 3120074 Total Pages: 41

Key Words: Year 2000. Y2K. Tank Waste Remediation System. TWRS, Procedure, Review. Report

Abstract: $N / A$

TRADEMARK DISCLAIMER. Reference herein to any specific comercial product, process, or service by trade name, trademark, manufacturer, or otherwise, does not necessarily constitute or imply its endorsement, recommendation, or favoring by the United States Government or any agency thereof or $i$ ts contractors or subcontractors.

Printed in the United States of America. To obtain copies of this document, contact: WHC/BCS Document Control Services, P.0. Box 1970, Mailstop H6-08, Richland WA 99352, Phone (509) 372-2420; Fax (509) 376-4989.
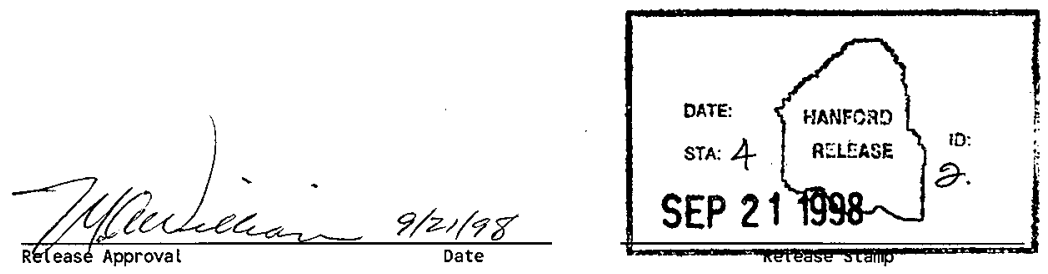


\section{Y2K TWRS MAINTENANCE PROCEDURE REVIEW:}

A concern exists that some equipment in use might contain microprocessors that are dependent upon a time date function. The majority of the software programming for microprocessors has only utilized a 2 digit identifier for the year. With the approach of the year $2000,(\mathrm{Y} 2 \mathrm{~K})$, there is concern that the date function will not be correctly recognized and some functions will not operate properly. TWRS maintenance procedures have been reviewed to identify equipment components that may not be Y2K compliant. Engineering judgement was utilized to eliminate procedures and equipment that is obviously not impacted by Y2K. (ex. W22036, Inspection of overhead doors; W22019, Inspection and test of GFCI receptacles; etc.)

The TWRS Maintenance Procedure index was utilized as a basis for reviewing the procedures. Initially, the index was reviewed and procedures dealing with equipment that was obviously compliant were identified. From that point, the procedures for suspect equipment were reviewed to determine if there was anything in the procedure to indicate existence of a time-date dependent function. Additionally, a computerized word search of the TWRS procedures was conducted to identify procedures containing the following key words:

\begin{tabular}{|l|l|l|}
\hline \multicolumn{3}{|c|}{ TWRS Maintenance Procedure Search: Y2K Key Words } \\
\hline Clock & LDK & Processor \\
\hline Computer & ENRAF & Timer \\
\hline PLC & Programmable & Micro-processor \\
\hline PCU-1 & TMCS & Controller \\
\hline Programmable Logic & Modicon & PAL-AT \\
\hline
\end{tabular}

A number of the procedures appeared as multiple "hits" on several of the search lists. Although identified in the search, often the key word was only used to identify associated equipment or functions and the equipment itself is not impacted by Y2K concerns. In other cases, the key word is used to describe a purely mechanical function that is not impacted by Y2K (ex. Clock or timer referring to a mechanical clock or timer ). Results of the search have been summarized as attached.

Following search of the procedures, the JCS was searched using procedure number to attempt to further determine if the equipment in question was Y2K compliant. From the JCS system, reference information such as CVI file and drawing numbers were obtained to permit additional research into the equipment or components.

Comments related to procedures for potentially suspect equipment have been identified on the Procedure Index. Additional research will be performed to determine compliance of the component. 


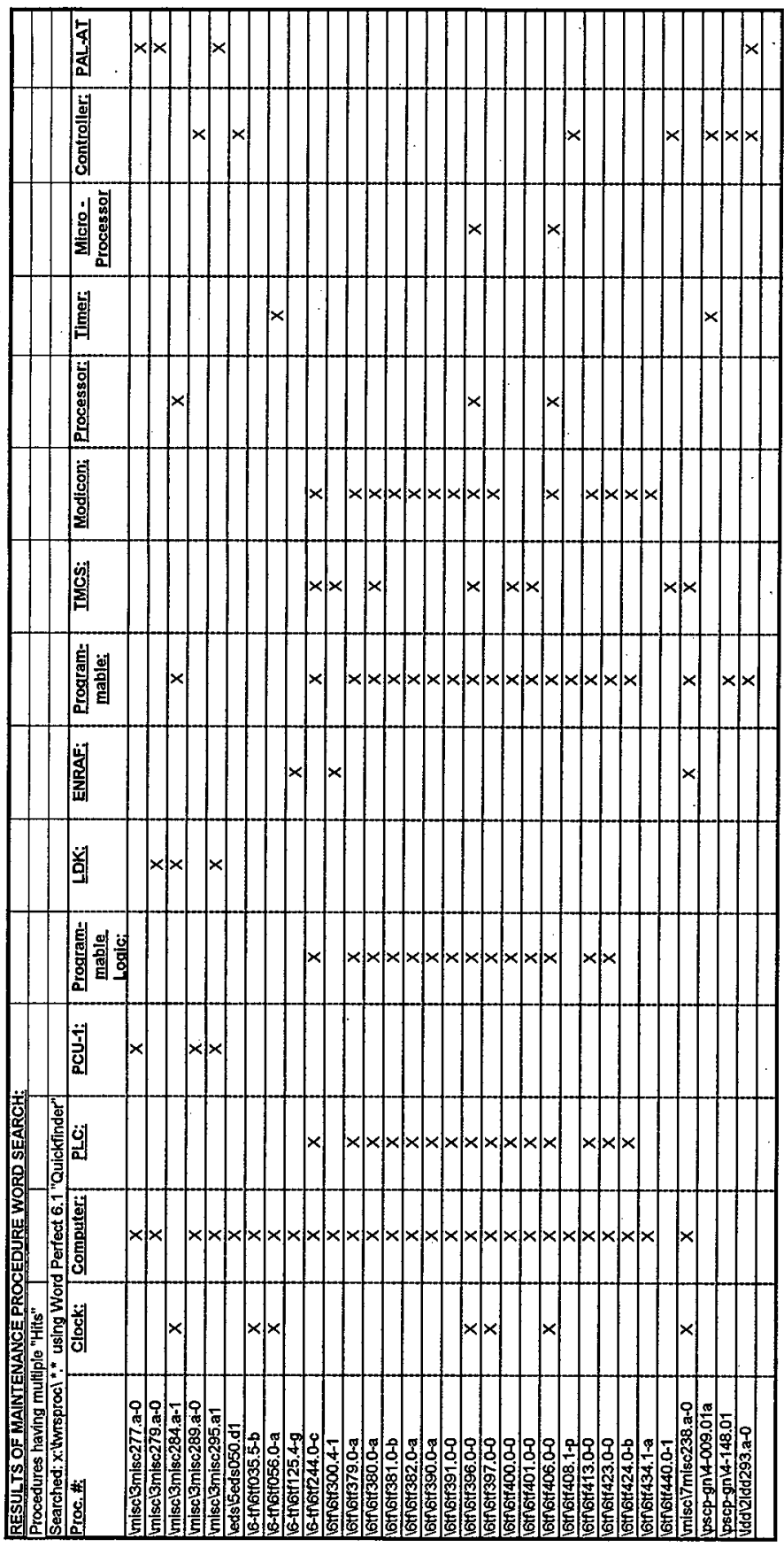



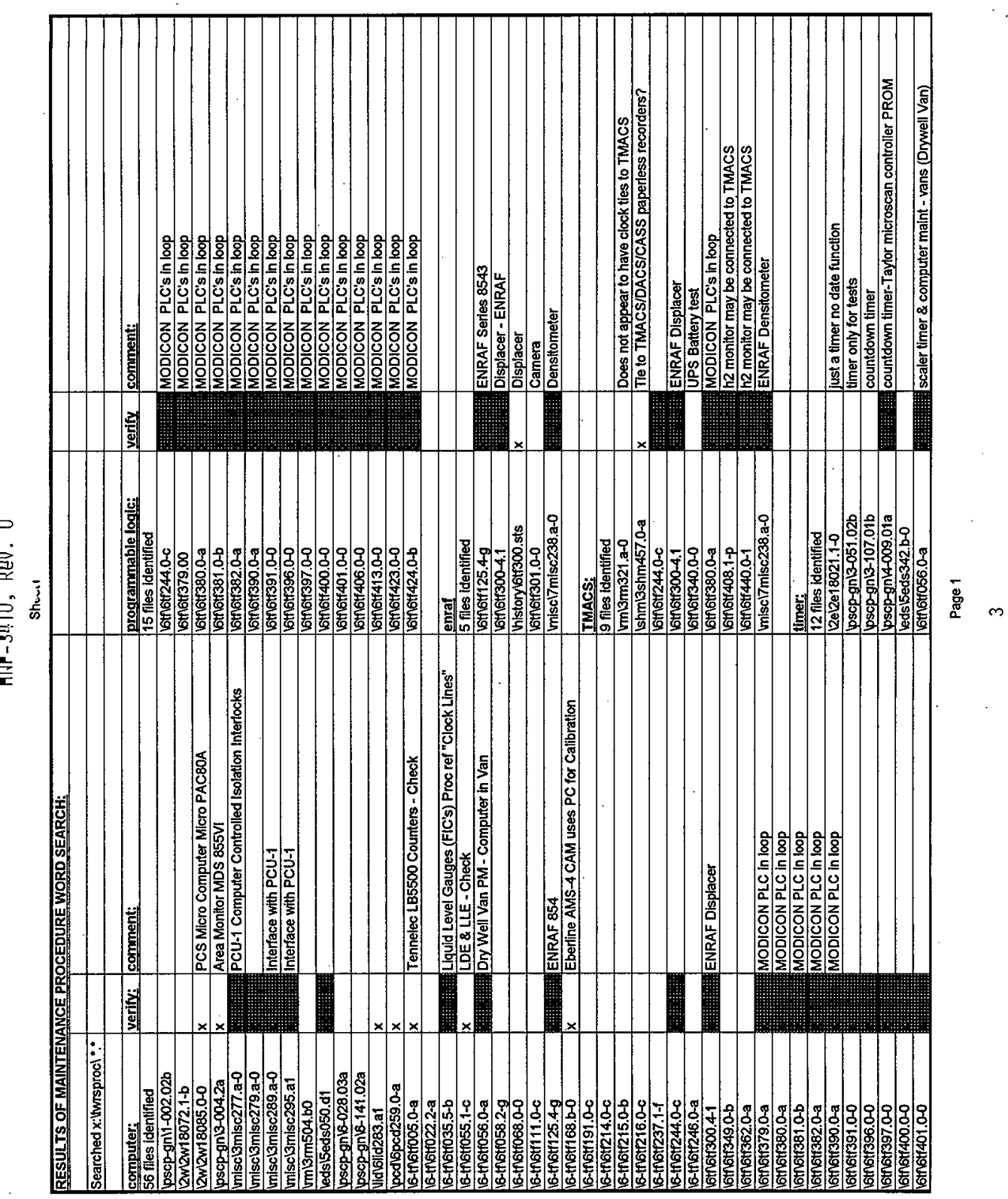


$$
\text { Simu1 }
$$

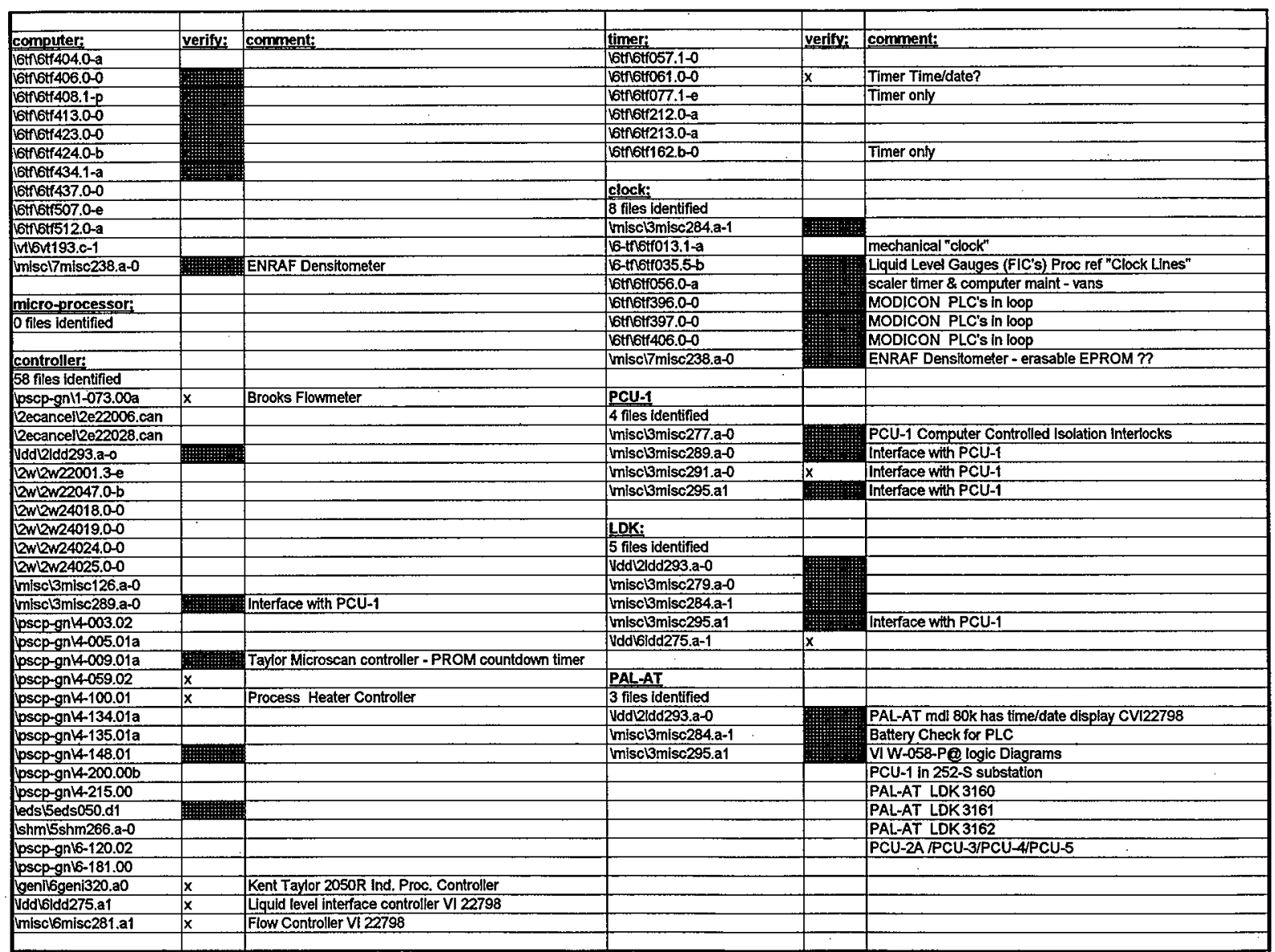

Page 2 
HINF-3410, Rev. U

Situdi

\begin{tabular}{|c|c|c|c|c|c|}
\hline controller: & verify: & comment: & processor: & verify: & comment: \\
\hline Imiscl6misc394.a0 & $x$ & & 5 files identified & & \\
\hline recd 16 recd $122 . \mathrm{b}-0$ & $x$ & & Vmiscl3misc284.a-1 & & Battery Check for PLC \\
\hline ted16ted312.b-0 & $x$ & & & & \\
\hline Ledi6tcd322.a0 & $x$ & & 6tn6ti387.0-0 & & Electronic high Bypass filter \\
\hline tedletcd323.a0 & $\bar{x}$ & & V6tn6tf396.0-0 & 雨 & MODICON PLC's in loop \\
\hline $16 t+6 t 010.1-a$ & & & Vetn6tf $405.0-0$ & $x$ & strain signal proc VI22671 MODICON PLC's \\
\hline 6ิtก6:f011.2-b & & & 16tnotf406.0.0 & & MODICON PLC's in loop \\
\hline \multicolumn{6}{|l|}{ Vistorylotfo11.sts } \\
\hline 6 tit 6 tfo52.0-b & & & micro processor: & & \\
\hline 16tibtf156tr.a-0 & & & 2 files identified & & \\
\hline Gtfott156tv.0-b & & & 1616 61396.00 & & MODICON PLC's in loop \\
\hline 16tfotf155tw.0-b & & & Wethotf $406.0-0$ & $\because$ & MODICON PLC's in loop \\
\hline \multicolumn{6}{|l|}{ 16tก6t:247.b-0 } \\
\hline 16 troti336.0-b & & & PLC'S: & & \\
\hline 16tfotf403.0-0 & & & 15 files ldentified & & \\
\hline bettotf408.1-p & & & 16tnotf244.0-c & & MODICON PLC's in loop \\
\hline btfotf427.0-0 & & & lotnotf379.00 & & MODICON PLC's in loop \\
\hline $16 t+60 t f 431.0-0$ & & & bin $6 t f 380.0-a$ & & MODICON PLC's in loop \\
\hline btf $6 t f 433.0-0$ & & & lotnotf38t.0-b & & MODICON PLC's in loop \\
\hline Wtfotf436.0-0 & & & 16tn6tf382.0-a & & MODICON PLC's in loop \\
\hline Whistory6tf436.sts & & & 16tก6tf390.0-a & & MODICON PLC's in loop \\
\hline เ.tกotf $439.0-0$ & & & 16tnotf39t.0-0 & m+口, & MODICON PLC's in loop \\
\hline Wtnotf440.0-1 & & & Votnotf396.0-0 & & MODICON PLC's in loop \\
\hline 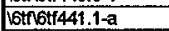 & & & Vetnotf397.0-0 & & MODICON PLC's in loop \\
\hline Vhistory6tf441.sts & & & $6 \ln 6 \mathrm{tf} 400.0-0$ & & MODICON PLC's in loop \\
\hline Ltfoth1515.0-0 & & & 16in6tf 401.00 & & MODICON PLC's in loop \\
\hline intovt245.b-0 & & & lotnotf 406.0 .0 & & MODICON PLC's in loop \\
\hline Iyt16ut454.a-0 & & & Wotnotf413.0-0 & 册 & MODICON PLC's in loop \\
\hline pscp-gni7-022.02 & & & 16tingtf $423,0-0$ & & MODICON PLC's in loop \\
\hline & & & $16 t 76 t f 424.0-b$ & : & MODICON PLC's in loop \\
\hline \multicolumn{6}{|l|}{ logic controllers: } \\
\hline \multirow[t]{2}{*}{0 files identified } & & & MODICON: & & \\
\hline & & & 19 files identified & & \\
\hline programmable: & & & 16tingtf244.0-c & & MODICON PLC's in loop \\
\hline 21 files identified & & & $16 \operatorname{tn} 6 t+371.00$ & $?$ & \\
\hline Ndd2ldd293.a-0 & & PAL-AT mdl b0k - has time/date function & 16tก6t3379.00 & & MODICON PLC's in loop \\
\hline unisc13misc284.a-1 & & & Gtnotr $380.0-a$ & & MODICON PLC's in loop \\
\hline pscp-gni4-148.01 & & & 6tnotf381.0-b & & MODICON PLC's in loop \\
\hline pscp-gni4-284.00a & & & lotnotr382.0-a & & MODICON PLC's in loop \\
\hline 16tก6tf244,0-c & & MODICON PLC's In loop & Votfotf $390.0-a$ & & MODICON PLC's in loop \\
\hline 6tก6t\$379.00 & & MODICON PLC's in loop & 6trnotr391.0-0 & & MODICON PLC's in loop \\
\hline 6tfotf380,0-a & & MODICON PLC's in loop & Vetfotr396.0.0 & & MODICON PLC's in loop \\
\hline Ltflot $381,0-b$ & (:) & MODICON PLC's in loop & Vetfotf397.0-0 & 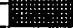 & MODICON PLC's in loop \\
\hline 6trut\}382.0-a & & MODICON PLC's in loop & Letnotf398.0-0 & & \\
\hline 6tกตt?390,0-a & 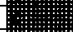 & MODICON PLC's in loop & lotfotf $400,0-0$ & & MODICON PLC's in loop \\
\hline Letfotf391,0-0 & 薄 & MODICON PLC's in loop & Gtfotf $401.0-0$ & & MODICON PLC's in loop \\
\hline 6t+16t/396.0-0 & 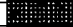 & MODICON PLC's in loop & Vtf 6 tf $406.0-0$ & 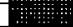 & MODICON PLC's in loop \\
\hline 5ิ+ก6t+397.0-0 & 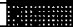 & MODICON PLC's in loop & 16tf $6 t f 408,0-0$ & & \\
\hline Gtfotf $400.0-0$ & 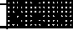 & MODICON PLC's in loop & Vtnotf413.0-0 & & MODICON PLC's in loop \\
\hline
\end{tabular}

Page 3 
HNt-34IU, ReV. U

Shieet1

\begin{tabular}{|c|c|c|c|c|c|}
\hline & & & & & \\
\hline programmable: & verify: & comment: & MODICON; & verify: & comment: \\
\hline wtก6ti $401.0-0$ & & MODICON PLC's in loop & fintet $423.0-0$ & & MODICON PLC's in loop \\
\hline $16 t \pi 6 t+406.0-0$ & & MODICON PLC's in loop. & $16 t \pi 6 t f 424.0-b$ & 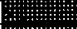 & MODICON PLC's in loop \\
\hline 16tfott $413.0-0$ & 粗 & MODICON PLC's in loop & 16 tribt4334.1-a & +10 & No clock - but tied to MODICON PLC \\
\hline $16 t$ tht $423.0-0$ & & MODICON PLC's in loop & & & \\
\hline 6tกตt(424,a-b & 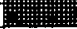 & MODICON PLC's in loop & & & \\
\hline $16 t$ fott $445.0-0$ & $x$ & Check VI Probably No time/date & & & \\
\hline miscl7misc238.a-0 & & ENRAF Densitomeler - erasable EPROM 7 & & & \\
\hline & & & & & \\
\hline
\end{tabular}




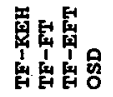

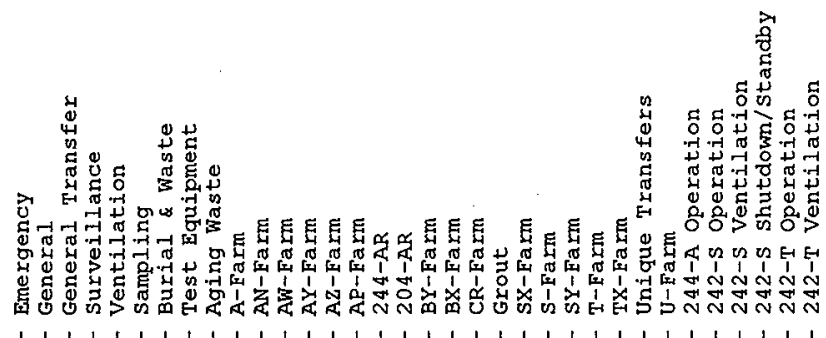

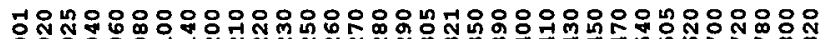

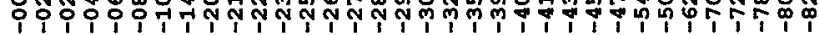

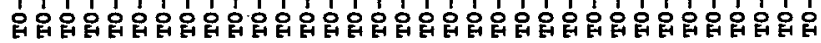

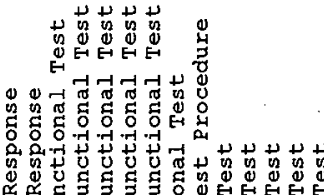

$\stackrel{\Downarrow}{n}$

o

o

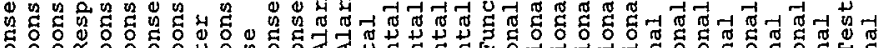

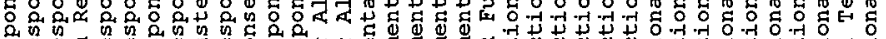

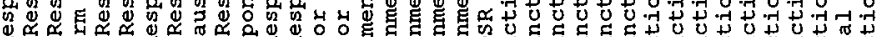

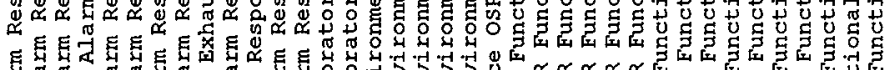

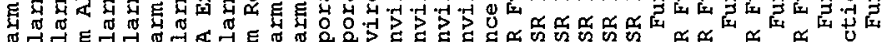

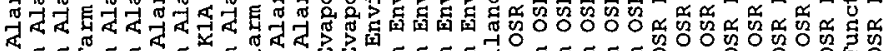

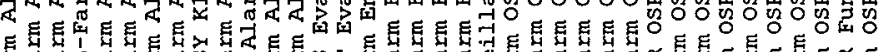

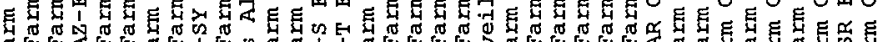

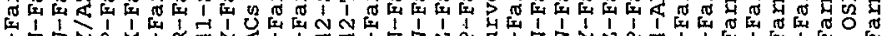

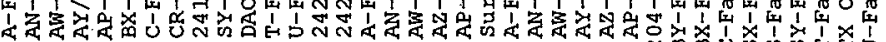

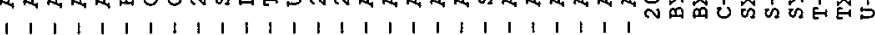

\section{on 0 웅}

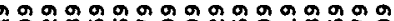

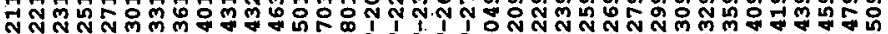
NTN

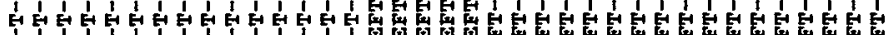

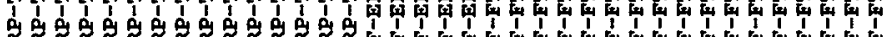

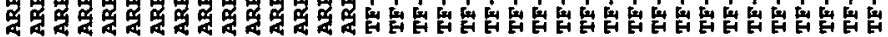




\section{Maintenanc Procedures}

1 - Loop Calibration

2 - Maintenance (Troubleshoot and or Repair)

3 - Functional Test

5 - Preventive Maintenance

6 - Calibration

4 - Predictive Maintenance

7 - General (Doesn't fit anything else)

BCDE = SYSTEM/COMPONENT DESIGNATOR

\begin{tabular}{|c|c|c|c|c|c|}
\hline $\mathrm{BCDE}$ & SYSTEM/COMPONENT DESIGNATOR & $\mathrm{BCDE}$ & SYSTEM/COMPONENT DESIGNATOR & $\mathrm{BCDE}$ & SYSTEM/COMPONENT DESIGNATÓR \\
\hline ABS & Asbestos & & Hydraulic system & VTA & Ventilation Tank Annulus \\
\hline ANN & Annunciator & IVS & Installed Vision systems & VTP & Ventilation Tank Primary \\
\hline $\mathrm{BA}$ & Breathing Air & ICD & Level Control Indicating Devices & WST & Waste Storage Tank \\
\hline CATH & Cathodic Protection & LDD & Leak Detectors and LDE devices & WSTA & Waste Storage Tank Annulus \\
\hline CASS & $\begin{array}{l}\text { Computex Automated } \\
\text { Surveillance System }\end{array}$ & LOV & Liquid Observation Vans & WT & Waste Transfer \\
\hline $\mathrm{CW}$ & Cooling water & MISC & Miscellaneous & & \\
\hline CVT & Converter/Transmitter & PCD & Pressure Control/Indicating Devices & & \\
\hline EBA & Emergency Breathing Air & RECD & Recorder or associated devices & & \\
\hline EDS & Electrical Distribution & RM & Radiation Monitoring & & \\
\hline EGEN & Generators Electrical & SA & Service Air & & \\
\hline EMER & Emergency System & SALW & Saltwell Equipment & & \\
\hline FACE & Facility/Shop Equipment & SHM & Standard Hydrogen Monitoring & & . \\
\hline $\mathrm{FCD}$ & $\begin{array}{l}\text { Flow Controller/Indicator } \\
\text { Device }\end{array}$ & TCD & $\begin{array}{l}\text { Temperature Control/Indicating } \\
\text { Devices }\end{array}$ & & \\
\hline GENI & General Instrument & TMAC & Tank Monitoring \& Control system & & \\
\hline GC & Gas Characterization & VAC & vacuum system & & \\
\hline GLY & Glycol Cooling & VB & Vent and Balance & & \\
\hline GM & Gas Monitoring & VT & Ventilation system & & \\
\hline
\end{tabular}

HNF-3410, Rev. 0 
PROCEDURE NUMBER

1-GENI-404

1-LDD-061

2-LCD-035

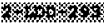

2-MAS-040

2-MISC-001

2-MISC -013

2-MISC-014

2-MISC-049

2-MISC-288

2-VT-262

2-WT-276

2E18021

$2 \Sigma 22003$

2E22020

2E22025

2E22039

2E22050

2E22091

2E22096

2E23032

2E24009

2E24022

2E 88004

\section{REV REV PROCEDURE TITLE}

B 0 INDEEENDENT SEEED/AMPS MONITORS 10I-SY LOOR CALIBRATION CHECKS

B $O$ CALIBRATION OF HORIZONTAL LATERAL MONITORING SYSTEM

G 0 TROUBLESHOOTING AND REPAIR OF FOOD INSTRUMENT CORPORATION LIQUID LEVEL GAU

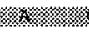

\% 0

SET-UP CONTINUOUS LEAK DETECTION SYSTEM FOR CROSS-SITE TRANSFERSO

TROUBLESHOOT AND REPAIR ANMUNCIATOR PANELS IN THE TANK EARM FACILITIES, 20

B 0 HANDLING AND HOOKUP OF TANK FARMS PUMPS

B 0

c 0

B 0

A 0

A 1

10

3 E

MAINTENANCE OF SPARLING METER, SPARLING 37770, HIGH TENSILE X-290

TANK FARM RUN-IN TEST FOR SUBMERSIBLE. AND FLYGT PUMPS

BOLT TORQUING GUIDELINES

PONDS/DITCHES/SLIDE GATES

REPLACEMENT OF CONSUMABLE COMPONENTS FOR BELT DRIVEN FANS AT EAST AREA TAN

DISASSEMBLY AND REASSEMBLY OF SOLENOID OPERATED VALVES IN THE DIVERSION BO

241-A-401 EAGLE TIMER RELAY FUNCTIONAL TEST

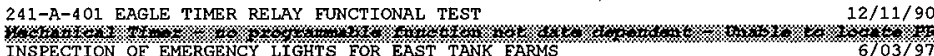

PREVENTIVE MAINTENANCE/ADJUSTMENTS FOR CASS MODEMS AND PMPLIEIER IN 200 AR $4 / 16 / 91$

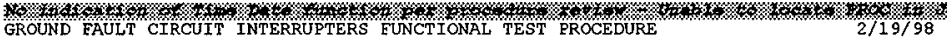

2750-E CASS POWERBLOC POWER CONDITIONING SYSTEM

FUNCTIONAL TEST OE CASS ALARMS FOR POWER CONDITIONING SYSTEM 2750-E BUILDI 11/16/95

SEMI-ANNUAL AIR COMPRESSOR INSPECTION 204-AR ( $\# 1$ )

CONDUCT VISUAL EXTERNAL INSPECTION OF SODIUM HYDROXIDE (NAOH) TANK TRUCK $6 \quad$ 8/11/92

PODS/DITCHES/SLIDE GATES

RELEASE DATE STATUS

7/15/98

$6 / 03 / 97$

$2 / 19 / 98$

$2 / 19 / 98$

$12 / 23 / 97$

$2 / 19 / 98$

$5 / 22 / 97$

$9 / 30 / 97$

$7 / 13 / 89$

REVISING

REVISING

ACT IVE

REVISING

REVISING

REVISING

REVISING

REVISING

REVISING

DEVELOPING

ACTIVE

ACTIVE

ACTIVE

ACTIVE

ACTIVE

ACTIVE

ACTIVE

ACTIVE

ACTIVE

ACTIVE

ACPIVE

ACTIVE

ACTIVE

HNF-3410, Rev. 0 
PROCEDURE NUMBER REV REV\# RROCEDURE TITLE

RELEASE DATE

STATUS

\%.

QREVENTIVE MAINTENANCE ACOUSTIC PROBE FOR DRY WELL VANS

RELEASE DATE

$6 / 29 / 90$

ACTIVE

$\mathrm{H}$

INSPECTION OF DRY WELT VAN PROBE CONECTIONS AND BOOH

INSE TON DNY 11/07/95

DRY WELL VAN COMPUTER BATTERY BACKUT FUNCTIONAL TTET

ACTIVE

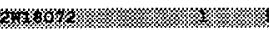

2W18080

$1 \quad A$

等

$4 / 24 / 96$

ACTIVE

Hzoros

INSPECTION AND CLEANING OF THE PCS MICRO COMPUTER (MICRO PAC 80/A)

$11 / 25 / 92$

$2 \mathrm{~W} 22001$

NA

720798

$5 / 02 / 95$

$7 / 15 / 98$

INSPECTION AND TEST OF GFCI RECEPTACLES

$2 / 23 / 98$

ACTIVE

2 W22019

PREVENTIVE MAINTENANCE AND FUNCTIONAL TEST OE 1000 CFM PORTABLE EXHAUSTER

$2 / 23 / 98$

ACTIVE

$2 \mathrm{~W} 22022$

INSPECTION OF OVERHEAD DOORS

200 WEST TANK FARMS DE-WINTERIZATION

$5 / 30 / 97$

PREVENTIVE MAINTENANCE OF LEAK DETECTOR CAISSON BLOWERS AND HOISTING MECHA

$6 / 16 / 89$

$6 / 29 / 90$

RCTIVE

$2 \$ 22047$

2W23007

2พ23008

2พ24011

2W24016

2W24018

2W24019

2W24022

2W24023

2พ24024

2W24025

2W24026

2W35004

3-EDS-081

PEDESTAL AND BENCH GRINDER INSPECTION - WEST MAINTENANCE SHOPS

$10 / 14 / 92$

ACTIVE

ACTIVATION ON "WINTERIZATION" 200 WEST TANK FARMS AND FACILITIES

$8 / 03 / 93$

RCTIVE

INSPECT AIR CONDITIONERS FOR DIGITAL ANALOG CONTROL SYSTEM (DACS) TRAILER

$7 / 23 / 94$

RCTIVE

ANNUAL MAINTENANCE FOR 241-SX-701 SAHARA EP-70 REGENERATIVE AIR DRYER

$7 / 23 / 94$

RCTIVE

MONTHLY MAINTENANCE FOR 241-SX-701 SAHARA EP-70 REGENERATIVE AIR DRYER

$7 / 23 / 94$

ACTIVE

AMNUAL MAINTENANCE FOR 241-U-701 HANKISON DH-45 REGENERATIVE AIR DRYER

$7 / 23 / 94$

ACTIVE

MONTHLY MAINTENANCE FOR 241-U-701 HANKISON DH-45 REGENERATIVE AIR DRYER

$7 / 23 / 94$

ACTIVE

ANNUAL MAINTENANCE FOR 242-S SAHARA EP-140 REGENERATIVE AIR DRYER

$7 / 23 / 94$

ACTIVE

MONTHLY MAINTENANCE FOR 242-S SAHARA ER-140 REGENERATIVE AIR DRYER

$7 / 23 / 94$

ACTIVE

ANNUAL MAINTENANCE FOR 241-T-601 TRINITY REGENERATIVE AIR DRYER

$1 / 05 / 93$

ACIIVE

INSPECT 272-WA MONORAIL HOIST (WH-03) WIRE ROPE

INSPECTION AND TEST OF GFCI RECEPTACLES

ACT IVE

ACT IVE

ACTIVE

ACTIVE

ACT IVE

ACT IVE

ACT IVE

REVISING

HNF-3470, Rev, 0 


\begin{tabular}{|c|c|c|c|c|c|}
\hline PROCEDURE NUMBER & REV & REV & PROCEDURE TITLE & RELEASE DATE & STATUS \\
\hline $3-E D S-180$ & $\mathbf{B}$ & 0 & GROUND FAULT CURRENT INTERRUPTERS FUNCTIOANL TEST PROCEDURE & & REVISING \\
\hline 3-EMER-316 & B & 0 & INSPECTION OF EMERGENCY LIGHTS FOR EAST TANK FARMS & & REVISING \\
\hline 3-EMER-317 & B & 0 & INSPECTION OF EMERGENCY LIGHTS FOR WEST TANK FARMS & $5 / 21 / 98$ & ACTIVE \\
\hline 3-LDD-053 & A & 0 & INTRINSICALLY SAFE LEAK DETECTOR RELAY BENCH TEST & & DEVELOPING \\
\hline $3-\mathrm{LDD}-055$ & A & 0 & TROUBLESHOOTING AND REPAIR OF LIQUID DETECTOR (LEAK DETECTOR ELEMENT AND L & & REVISING \\
\hline 3-LDD-058 & D & 0 & VERIFICATION OF FOOD INSTRUMENT CORPORATION LIQUID LEVEL GAUGE REFERENCE E & & REVISING \\
\hline $3-\mathrm{MISC}-126$ & A & 0 & EAST TANK FARMS WEEKLY CHECK OF HEATER AND HEAT TRACE & $1 / 09 / 98$ & ACTIVE \\
\hline \% & 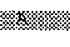 & & FUNCTIONAL CHECK OF REPLACEMENT CST SYSTEM (W-058) TRANSFER PUMP SHUTDOWN & $3 / 06 / 98$ & ACTIVE \\
\hline 3-MTSC-279 & A & 0 & $\begin{array}{l}\text { FUNCTIONAL TEST OE REPLACEMENT CROSS-SITE TRANSEER SYSTEM BOOSTER PUMP SHU } \\
\text { :H }\end{array}$ & $3 / 12 / 98$ & ACT IVE \\
\hline 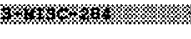 & 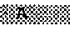 & 榤 & FUNCTIONAL CHECK OF THE PAL-AT BACKUP BATTERY & $4 / 03 / 98$ & ACT IVE \\
\hline 3-MISC-289 & 0 & 0 & FUNCTIONAL CHECK OF REPLACEMENT CROSS SITE TRANSFER SYSTEM $(W-058)$ ELUSH S & $3 / 07 / 98$ & ACTIVE \\
\hline 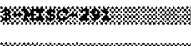 & \%x: & & 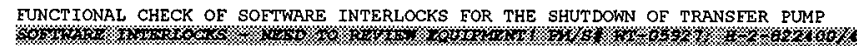 & $3 / 07 / 98$ & ACT IVE \\
\hline $3 \mathrm{x}$ & 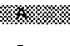 & 陊然 & FUNCT IONAL CHECK OF CROSS-SITE TRANSFER LINE LEAK DETECTION COMPUTER GENER & $4 / 03 / 98$. & ACTIVE \\
\hline 3-MISC -296 & A & 0 & FUNCTIONAL CHECK OF REPLACEMENT CROSS SITE TRANSEER SYSTEM BOOSTER PUMP SH & & DEVELOPING \\
\hline $3-M x S C-363$ & A & $\mathbf{0}$ & WINTERIZATION/DE-WINTERIZATION EAST TANK FARMS & $10 / 16 / 97$ & ACTIVE \\
\hline $3-\mathrm{RM}-321$ & A & 0 & CALIBRATE NCR MODEL ADM-61OA RADIATION INDICATOR WITH MODEL GP-100C RADIAT & $6 / 09 / 98$ & ACTIVE \\
\hline $3-R M-504$ & $\mathrm{~B}$ & 0 & 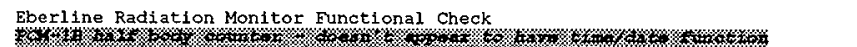 & $9 / 11 / 97$ & ACTIVE \\
\hline 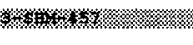 & 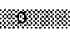 & & $\begin{array}{l}\text { WHITAKER HYROAGEN CELI FUNCTIONAL TEST } \\
\text { W }\end{array}$ & $2 / 24 / 98$ & ACTIVE \\
\hline $3-\sqrt{8}-157 A$ & $\mathbf{B}$ & 0 & APPENDIX A $241-A$ TANK FARM BREATHER FILTER AEROSOL TEST DATA SHEETS & & REVISING \\
\hline $3-V B-157 A X$ & $\mathbf{B}$ & 0 & APPENDIX AX, AX TANK FARM BREATHER FILTER AEROSOL TEST DATA SHEETS & & REVISING \\
\hline $3-v_{B}-157 B$ & B & 0 & APPENDIX B, 241-B TANK FARM BREATHER AEROSOL TEST DATA SHEETS & & REVISING \\
\hline $3-V B-157 \mathrm{BX}$ & B & 0 & APPENDIX BX, 241-BX TANK FARM BREATHER FILTER AEROSOL TEST DATA SHEETS & & REVISING \\
\hline $3-V B-157 \mathrm{BY}$ & $B$ & 0 & A.PENDIX BY, 241-BY TANK FARM BREATHER FILTER AEROSOL TEST DATA SHEETS & & REVISING \\
\hline $3-v B-157 C$ & B & 0 & APPENDIX $\mathrm{C}, 241-\mathrm{C}$ TANK EARM BREATHER EILTER AEROSOL TEST DATA SHEETS & & REVISING \\
\hline
\end{tabular}

HNF-3410, Rev. 0 


\begin{tabular}{|c|c|c|c|c|c|}
\hline PROCEDURE NUMBER & REV & REV\# & PROCEDURE TITLE & RELEASE DATE & STATUS \\
\hline $3-V B-157 D U$ & B & 0 & APPENDIX DU, 244-U DCRT BREATHER FILTER AEROSOL TEST DATA SHEETS & & REVISING \\
\hline $3-V B-157 E W$ & B & 0 & APPENDIX EW, 241-EW-151 TANK BREATHER FILTER AEROSOL TEST DATA SHEETS & & REVISING \\
\hline $3-V B-1570 B$ & $\mathbf{B}$ & 0 & APPENDIX QB, 241-BX-101 BREATHER FILTER AEROSOL TEST DATA SHEETS & & REVISING \\
\hline $3-\mathrm{VB}-157 \mathrm{QC}$ & B & 0 & APPENDIX QC, 241-C-103 BREATHER FILTER AEROSOL TEST DATA SHEETS & & REVISING \\
\hline $3-\mathrm{VB}-157 \mathrm{QT}$ & B & 0 & APPENDIX QT, 241-TX-113 BREATHER FILTER AEROSOL TEST DATA SHEETS & & REVISING \\
\hline $3-V B-1575$ & B & 0 & APPENDIX $S, 241-S$ TANK EARM BREATHER AEROSOL TEST DATA SHEETS & & REVISING \\
\hline $3-V B-1575 S$ & B & 0 & APPENDIX SS, 241-S-304 CATCH TANK BREATHER FILTER AEROSOL TEST DATA SHEETS & & REVISING \\
\hline $3-V B-1575 X$ & B & 0 & APPENDIX SX, 241-SX TANK FARM BREATHER AEROSOL TEST DATA SHEETS & & REVISING \\
\hline $3-V B-157 T$ & $\mathbf{B}$ & 0 & APPENDIX T, 241-T TANK FARM BREATHER AEROSOL TEST DATA SHEETS & & REVISING \\
\hline $3-V B-157 T X$ & B & 0 & APPENDIX TX, 241-TX TANK FARM BREATHER AEROSOL TEST DATA.SHEETS & & REVISING \\
\hline $3-V B-157 T Y$ & B & 0 & APPENDIX TY, 241-TY TANK FARM BREATHER AEROSOL TEST DATA SHEETS & & REVISING \\
\hline $3-V B-157 U$ & B & 0 & APPENDIX U, 241-U TANK FARM BREATHER AEROSOL TEST DATA SHEETS & & REVISING \\
\hline $3-\mathrm{VB}-157 \mathrm{VA}$ & B & 0 & APPENDIX VA, 6241-A CROSS SITE FACILITY BREATHER FILTER AEROSOL TEST DATA & & REVISING \\
\hline $3-\mathrm{VB}-157 \mathrm{VV}$ & $\mathbf{B}$ & 0 & APPENDIX VV, $6241-V$ CROSS SITE FACILITY BREATHER FILTER AEROSOL TEST DATA & & REVISING \\
\hline $3-\mathrm{VB}-157 \mathrm{~W}$ & B & 0 & APPENDIX $w$, 213-W RETENTION TANK BREATHER FILTER AEROSOL TEST DATA SHEETS & & REVISING \\
\hline $3-V B P-157$ & B & 0 & BREATHER FILTER IN-PLACE LEAK TEST (AEROSOL TEST) & & REVISING \\
\hline $5-\mathrm{BA}-124$ & B & 0 & PORTABLE BREATHING AIR COMPRESSORS (H0-64-3848 AND -3849) CARBON MONOXIDE & & REVISING \\
\hline 5-CATH-221 & C & 0 & INSPECTION OF CATHODIC PROTECTION SYSTEM RECTIEIERS & & REVISING \\
\hline 5-EDS-044 & B & 0 & 480-VOLT FEDERAL PACIFIC CIRCUIT BREAKER INSPECTION AND TESTING & & REVISING \\
\hline 5-EDS-050 & $\mathrm{D}$ & 1 & ELECTRIC MOTOR INSPECTION & $4 / 03 / 98$ & ACTIVE \\
\hline 5-EDS-146 & A & 0 & LOW VOLTAGE ELECTRICAL DISTRIBUTION SYSTEM INSEECTION AND TESTING & $2 / 27 / 98$ & ACTIVE \\
\hline $5-E D S-278$ & A & 1 & INSPECT AND TEST SWITCHBOARD SB-1 480-VOLT POWER GIRCUIT BREAKERS FOR REPL & $6 / 05 / 98$ & ACTIVE \\
\hline 5-EDS -341 & B & 0 & INTERNATIONAL POWER MACHINES, MODEL IBP+10 UNINTERRUPTABLE POWER SUPPLY, $c$ & $10 / 31 / 97$ & ACTIVE \\
\hline $5-E D S-342$ & B & 0 & AUTO-TRANSEER SWITCH, ZENITH CNTL ZTSH SERIES INSP & $10 / 31 / 97$ & ACTIVE \\
\hline
\end{tabular}

$$
\text { HNF-3410, Rev. } 0
$$




\begin{tabular}{|c|c|c|c|c|c|c|}
\hline \multicolumn{2}{|c|}{ PROCEDURE. NUMBER } & REV & REV\# & RROCEDURE TITLE & RELEASE DATE & STATUS \\
\hline 5-EDS-383 & & $\mathrm{B}$ & 0 & NEED TITLE & & REVISING \\
\hline 5-EGEN-063 & & B & 0 & HONDA ES- 6500 GENERATOR PREVENTIVE MAINTENANCE & ' & REVISING \\
\hline $5-E G E N-332$ & & B & 0 & AY/AZ VENTILATION AND COOLING STANDBY GENERATOR MONTHLY PREVENTIVE MAINTEN & & REVISING \\
\hline 5-EMER-062 & & B & 0 & REMOTE EMERGENCY SIREN LOCAL CLEANING AND INSPECTION & & REVISING \\
\hline 5-EMER-089 & 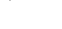 & B & 0 & INSPECTION AND CLEANING OF EXIT LIGHTS FOR WEST TANK FARMS & & REVISING \\
\hline 5-EMER-194 & & 1 & D & FIRE DOOR INSPECTION & $5 / 18 / 98$ & ACTIVE \\
\hline 5-EMER-366 & & B & 0 & Encon Portable Eyewash Stations, Inspection/Maintenance & & REVISING \\
\hline 5-FACE -330 & & B & 0 & LOAD TEST 272-AW MONORAIL HOIST (EH-01) & & REVISING \\
\hline 5-MISC-127 & & A & 0 & PEDESTAL AND BENCH GRINDER INSPECTION - WEST MAINTENANCE SHOPS & & DEVELOPING \\
\hline $5-\mathrm{MISC}-285$ & & A & 0 & INSEECT REPLACEMENT CROSS SITE TRANSFER SYSTEM AIR COMPRESSORS AND AIR DRY & $3 / 07 / 98$ & ACTIVE \\
\hline 5-MI SC-285 & & B & 0 & INSPECT REPLACEMENT CROSS SITE TRANSFER SYSTEM AIR COMPRESSORS AND AIR DRY & & REVISING \\
\hline 5-MI SC-297 & & C & 0 & INSPECTION OF OVERHEAD DOORS & & REVISING \\
\hline 5-MI SC-299 & & $\mathbf{E}$ & 0 & PORTABLE TOOLS AT 272-WR MAINTENANCE & & REVISING \\
\hline $5-\mathrm{MISC}-305$ & & C & 0 & DRY WELL VAN COMPUTER BATTERS BACKUP FUNCTIONAL TEST & & REVISING \\
\hline 5-MISC-306 & & D & 0 & INSPECTION OF DRY WELL VAN PROBE CONNECTIONS AND BOOM PENDANT SWITCH & & REVISING \\
\hline 5-MI SC-315 & & B & 0 & CONDUCT VISUAL INSPECTION OF SODIUM HYDROXIDE (NaOH) TANK TRUCK 68C-4347 & & REVISING \\
\hline $5-$ MISC- 331 & & C & 0 & ACOUSTIC PROBE FOR DRY WELL VANS & & REVISING \\
\hline 5-MI SC- 375 & & C & 0 & PREVÈNTIVE MAINTENANCE OF LEAK DETECTOR CAISSON BLOWERS AND HOISTING MECHA & & REVISING \\
\hline $5-S A-139$ & & A & 0 & INGERSOLL-RAND 200 HP AIR COMPRESSOR SEMI-ANNUAL MAINTENANCE & & DEVELOPING \\
\hline $5-\$ A-370$ & & B & 0 & SEMI-ANNUAL AIR COMPRESSOR INSPECTION - 204-AR (\#1) & & REVISING \\
\hline $5-S H M-266$ & & A & 0. & PREVENTIVE MAINTENANCE FOR HYDROGEN MONITORING SYSTEM GAS SAMPLE CONDITION & $5 / 28 / 98$ & ACTIVE \\
\hline $5-\mathrm{TCD}-307$ & & C & 0 & MANUAL TEMPERATURE READINGS FOR WEST TANK FARMS & & REVISING \\
\hline $5-V T P-076$ & & E & 0 & BREATHER FILTER REPAIR AND REPLACEMENT & & REVI SING \\
\hline 6 -CASS-313 & & E & 0 & FUNCTIONAL TEST OF CASS ALARMS FOR FOWER CONDITIONING SYSTEM - 2750E BUILD & & REVISING \\
\hline
\end{tabular}

HNF-3410, Rev. 0 
PROCEDURE NUMBER REV REV* PROCEDURE TITLE

6-CASS-314

RELEASE DATE STATUS

REVISING

$6-\mathrm{CVT}-329$

CALIBRATE EISHER-ROSEMOUNT MODEL 3044CA115M5 TEMPERATURE TRANSMITTER

$5 / 22 / 98$

ACTIVE

$6-\mathrm{CVT}-384$

BAILEY 7000 VOLTAGE TO FREQUENCY CONVERTER MODEL 741 CALIBRATION PROCEDURE

$6-\mathrm{CVT}-411$

ACROMAG SERIES 800 ENEUMATIC CONVERTERS CALIBRATION PROCEDURE

$6-\mathrm{CVT}-447$

$6-\mathrm{CVT}-472$

ROBERSHAW MODEL 443 AND 445 ELECTRO-PNEUMATIC CONVERTER CALIBRATION PROCED

$6-\mathrm{CVT}-474$

CALIBRATE DIFFERENTIAL PRESSURE TRANSMITTERS FOR 242A

$6-\mathrm{CVT}-476$

CALIBRATE ELECTRONIC PRESSURE AND DIFFERENTIAL PRESSURE TRANSMITTER LESLIE

$6-$ CVT -515

CALIERATE EMERSON-TYRE MAGNETIC FLOW SIGNAL CONVERTERS SERIES 7000

6-CVT-517

Ba1ley Model 740 Millivolt Converters

$6-C V T-519$

FAIRCHILD MODELS T-5100B-1. T-5100B-4, 5200 AND T-5600 I/P TRANSDUCERS CAL

$6-$ CVT -520

$6-$ CVT -527

$6-\mathrm{CVT}-538$

$6-\mathrm{FCD}-385$

$6-\mathrm{FCD}-481$

6-FCD-510

TELMAR, SERIES 500 MILLIVOLT-TO-CURRENT TRANSMTTTER CALIBRATION PROCEDURE

FOXBORO E69 SERIES CURRENT-TO-PNEUMATIC CONVERTERS AND POSITIONS CALIBRATI

E OR I/P CONVERTER CALIBRATION PROCEDURE

BROOKS MODEL 3560, SERIES III MAG-FLOW CONVERTERS CALIBRATION PROCEDURE

CALIBRATE AMC MASS TRON IT

CALIBRATE MARSH-MCBIRNEY, MODEL 250 FLOWMETER

REVISING

REVISING

REVISING

REVISING

REVISING

REVISING

REVISING

REVISING

REVISING

REVISING

6-GENI -010

AIR ROTOMETER CALIBRATION

6-GENI-131

FISHER CONTROL, CAGELESS 249 SERIES DISPLACER SENSOR MAINTENANCE

ACTIVE

$7 / 14 / 98$

REVISING

6-GENI-135

CALIBRATE ALARM MODULE

$5 / 12 / 98$

ACTIVE

REVISING

REVISING

REVISING

REVISING

GENERIC CALIBRATION OE DIGITAL INDICATORS

6-GENI-294

TAYLOR MIDEL 1015N AND 1016N ELETRONIC ALRAM CALIBRATION PROCEDURE

REVISING

REVISING

6-

6-GENI-324

CALIBRATE LEE MODEL $371-\mathrm{K}$ INDICATORS WITH CURRENT INPUT

$6-G E N I-325$

A 0

CALIBRATE TRIPLETT MODEL UB120-14-1-G TILT POSITION INDICATOR

$5 / 14798$

ACTIVE

ACTIVE

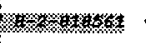

$5 / 12 / 98$ ACTIVE

HNF-3410, Rev. 0 
PROCEDURE NUMBER REV REV PROCEDURE TITLE

6-GENI-354

6-GENI-377 A

6-GENI-451 B 0

6-GENI-453 B 0

6-GENI-459 B 0

6-GENI-471 B 0

$6-G E N I-475 \quad B \quad 0$

$6-G E N I-478 \quad$ B

$6 \leftrightarrow$ GENI $-479 \quad$ B 0

6-GENI-480 B

6-GM-143 B 0

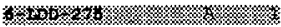

6-LDD-286

B $\quad 0$

6-LDD-319

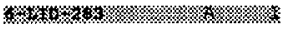

\$

6\%

6-MI SC-142

6-MI SC-28I

6-MI SC-282

6-MISC-292

6-MISC-298

$6-$ MI SC- 336

6-MISC-394
CALIBRATE KINEMETRICS MODEL TS-33MOD SEISMIC TRIGGER

PD690 DIGITAL PROCESS METER CALIBRATION

CALIBRATE LESLIE MODULE 610323L LIMIT ALARMS

TAYLOR INDICATOR AND INDICATING CONTROLLER, SERIES 1300 CALIBRATION PROCED

MOORE PRODUCTS SERIES 72 VALVE POSITIONER CALIBRATION

CALIBRATE MASONEILAN ELECTO PNEUMATIC TRANSDUCERS MODELS 8005 AND 8006

CALIBRATE YEW SERIES 80, MODELS SLPC AND SLCD RROGRAMMABLE INDICATING CONT

CALIBRATE BAILEY 7000 SINGLE POINT INDICATOR, MODEL 730

CALIBRATE TAYLOR MICRO-SCAN INDICATING CONTROLLER, 1300 SERIES

BRUEL \& KJAER 1302 MULTI-GAS MONITOR CALIBRATION

CALIBRATE THE DIVERSION BOX AND VENT STATION SUMP PUMP LEAK DETECTION ELEM

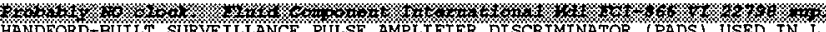

ROBERSHAW MODEL 185A (R) PRECISION GAUGE CALIBRATION PROCEDURE

FOXBORO IFL10-1

FOXBORO IFL10-1 3.1\%

4.

FOXBORO $760 \% 761$ SINGLE STATION

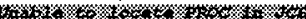

ROCHESTER INSTRUMENT SYSTEMS, SIGANL TRANSMITTER, MODEL SC-1300

CALIBRATION OF PANAMETRICS MODEL DF868 ULTRASONIC FLOW INDICATOR TRANSMITT

CALIBRATION OF THE EOXBORO MODEL IMT25 I/A SERIES MAGNETIC TRANSMITTERS FE

ADJUSTMENT FOR CASS MODEMS AND PMPLIFIER IN 200 AREA TANK EARMS

INSPECTION AND CLEANING OF THE PCS MICRO COMPUTER

AY/AZ CENTURA VALVE ALIGNMENT \& SERVO CALIBRATION

CALIBRATE AMC VEL-TROL II
RELEASE DATE STATUS

$7 / 21 / 98 \quad$ ACTIVE

$5 / 22 / 98 \quad$ ACTIVE

$5 / 27 / 98 \quad$ ACTIVE

REVISING

REVISING

REVISING

REVISING

REVISING

REVISING

REVISING

DEVELOPING
ACTIVE

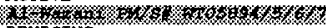

REVISING

REVISING

ACTIVE

REVISING

REVISING

REVISING

4/03/98 ACTIVE

4/03/98 ACTIVE

REVISING

REVISING

REVISING

5/22/98 ACTIVE

HNF-3410, Rev. 0 


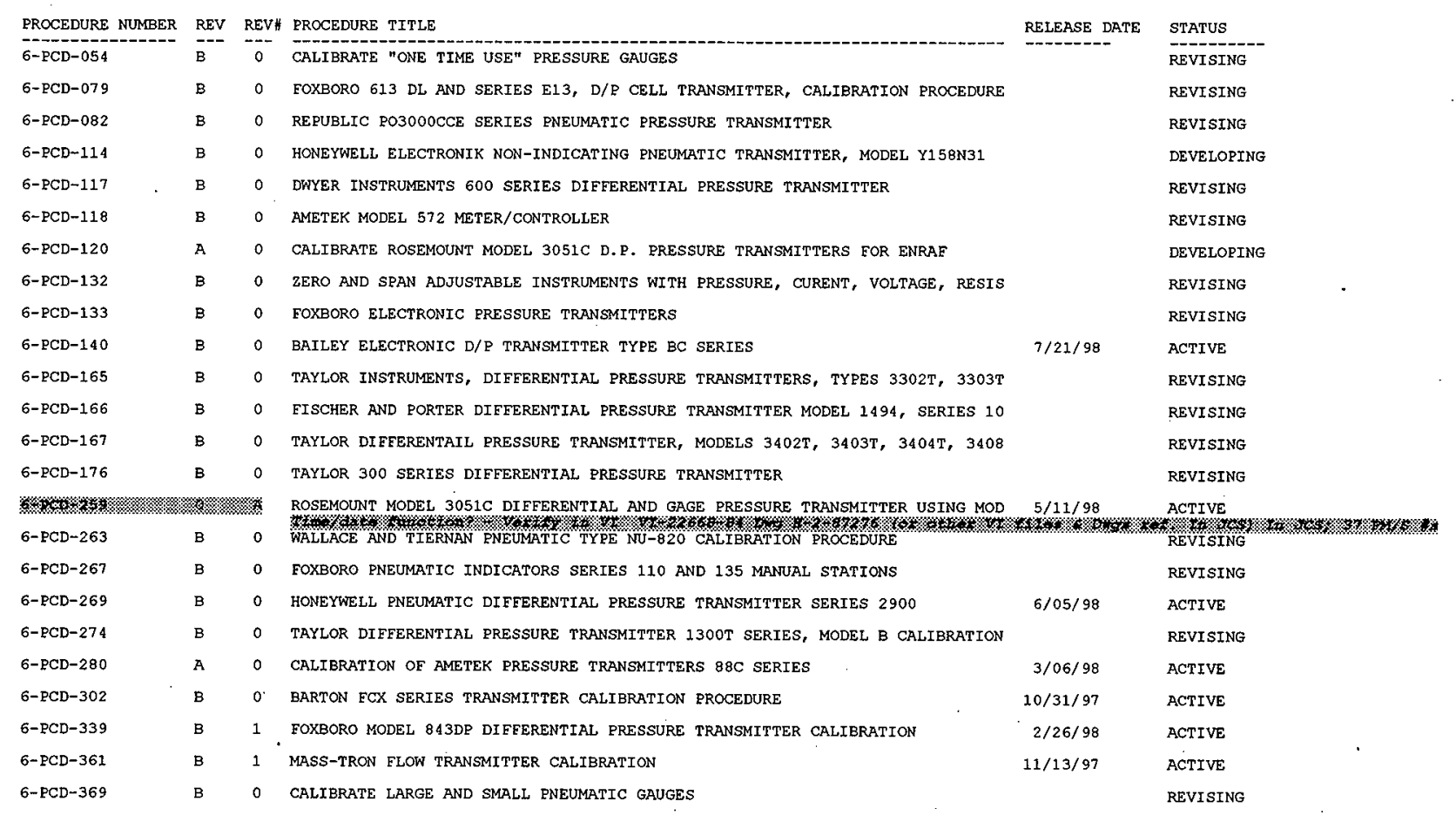

$$
\text { HNF-3410, Rev. } 0
$$




\begin{tabular}{|c|c|c|c|c|c|}
\hline PROCEDURE NUMBER & REV & REV" & PROCEDURE TITLE & RELEASE DATE & STATUS \\
\hline $6-\mathrm{PCD}-373$ & B & 0 & FOXBORO DIFFERENTIAL PRESSURE TRANSMITTERS SERIES $13,13 \mathrm{~A}, 13 \mathrm{H}$, AND 15 & & REVISING \\
\hline $6-\mathrm{PCD}-393$ & A & 0 & CALIBRATE BARTON MODEL 289A DIFFERENTIAL PRESSURE INDICATING SWITCH & $5 / 12 / 98$ & ACTIVE \\
\hline $6-\mathrm{PCD}-431$ & B & 0 & DIFFERENTIAL PRESSURE LOOP VERIEICATION TEST DATA SHEETS & & REVISING \\
\hline $6-P C D-458$ & A & 0 & CALIBRATE PRESSURE SWITCHES & & DEVELOPING \\
\hline $6-\mathrm{PCD}-460$ & B & 0 & WESTINGHOUSE PRESSURE TRANSMITTERS, MODELS 56PL, PM, AND PH CALIBRATION & & REVISING \\
\hline $6-\mathrm{PCD}-508$ & B & 1 & DIAPHRAGM OPERATED PRESSURE SWITCHES & $10 / 02 / 97$ & ACTIVE \\
\hline 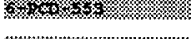 & $\%$ & 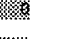 & 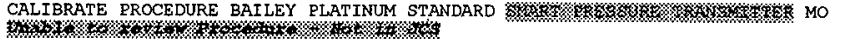 & & DEVELOPING \\
\hline $6 \mathrm{x}$ & 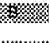 & 镐 & LEEDS ANN NORTHRUP SPEEDOOMAX G, H, AND W & & REVISING \\
\hline 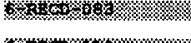 & ren & 4 & 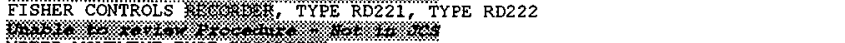 & & REVISING \\
\hline $6 \times 3 \times 8 \times 88$ & 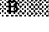 & 㜣 & 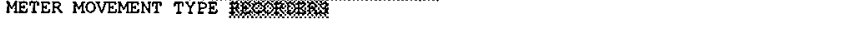 & - & REVISING \\
\hline \% & x) & 器 & 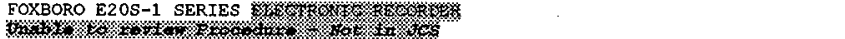 & & REVISING \\
\hline 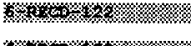 & Stan & 践 & FOXBORO MODEL 53 AND 54 CONSOTROL & $6 / 05 / 98$ & ACTIVE \\
\hline Sos & 4 & 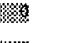 & 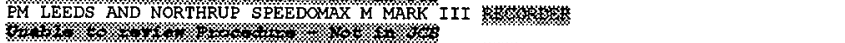 & & REVISING \\
\hline 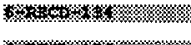 & 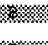 & \$? & 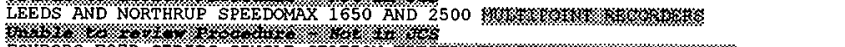 & & REVISING \\
\hline $6 x+40 \%$ & tow. & 好 & 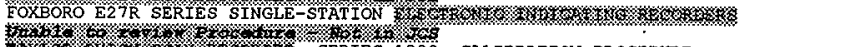 & & REVISING \\
\hline 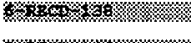 & t5. & 緗 & 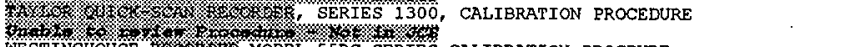 & & REVISING \\
\hline 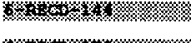 & 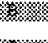 & & 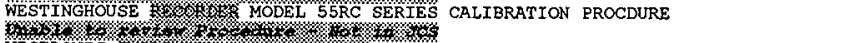 & & REVISING \\
\hline 6 & X). & & 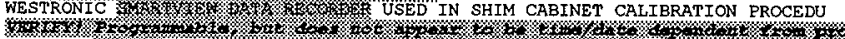 & $8 / 27 / 97$ & ACTIVE \\
\hline 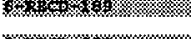 & 8 & 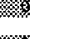 & FOXBORO & & REVISING \\
\hline W & $x$ & 然签 & YOKAGAWA (YEW) MICRO R100 SERIES U Y & $9 / 03 / 97$ & ACTIVE \\
\hline $38 \times x_{0}$ & \&\$ & 蝲 & 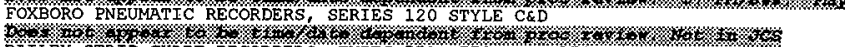 & $5 / 29798$ & ACTIVE \\
\hline $6-R E C D-364$ & B & 0 & BAILEY STRIP-CHART, RECORDERS, TYPES 771, 772, AND 773 & $7 / 21 / 98$ & ACTIVE \\
\hline$\% \%$ \% & x/x & & 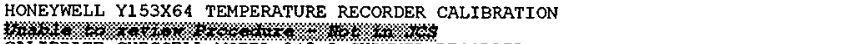 & & REVISING \\
\hline 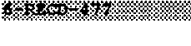 & 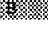 & & 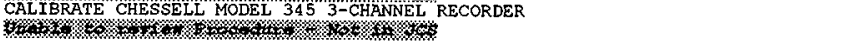 & & REVISING \\
\hline
\end{tabular}

HNF-3410, Rev. 0 
OCEDURE TITLE

CALIBRATE SAIC/RADECO, HI-Q ENVIRONMENTAL AND KM

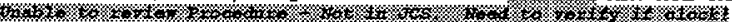

HARSHAW, MODEL NR-25, LOGARITHMIC-LINEAR RATEMETER, CALIBRATION PROCEDURE

CANBERRA LIN/LOG RATEMETER MODEL $1481 \mathrm{~L}$

ELECTRONICALLY CALIBRATE VICTOREEN, G-M AREA MONITORING SYSTEM, MODEL 855/

ROBERTSHAW, TR-1000 SERIES, TEMPERATURE REGULATING VALVE

WESTON MODEL 4303 AND 4503 DIAL THERMOMETERS CALIBRATION PROCEDURE

ACTION PAK INSTRUMENTS WITH RTD AND BRIDGE INPUT AND VOLTAGE/CURRENT OUTPU

ACTION MIDEL 4041 RTD DIFEERENTIAL TEMPERATURE TRANSMITTER CALIBRATION PRO

OMEGA SERIES HH-99A HAND HELD DIGITAL THERMOMETER

CALIBRATE LOVE CONTROLS TEMPERATURE ALARM SWITCH MODELS 48, 49, AND $50 \quad 7 / 14 / 98$

CALIBRATION PROCEDURE FOR TEMPERATURE SWITCH

RDE TEMPERATURE TRANSMITTER MODELS 2800 AND 2900 ADJUSTMENT AND CALIBRATIO

WASTE TANK MAINTENANCE PROCEDURE, FOXBORO MODEL 893RA-Q TEMPERATURE TRANSM.

WASTE TANK MAINTENANCE PROCEDURE WATLOW MODEL 988 TEMPERATURE CONTROLLER C

CALIBRATE CHROMALOX MODEL 2104 TEMPERATURE CONTROLLER

CALIBRATE CHROMALOX MODEL 3101-11000 OVERTEMPERATURE INDICATING SWITCH

CALIBRATE DEVAR MODEL SM-812/M371/18PIX TEMPERATURE TRANSMTTTER

CALIBRATE DEVAR MODEL 18-328 ALARM

CALIBRATE PYROMATION MODEL R1T185L TEMPERATURE TRANSMITTER

OMEGA DIGITAL TEMPERATURE INDICATOR, MODEL 199, CALIBRATION PROCEDURE

CHROMALOX 3914 EXHAUSTERS HEATER CONTROLS, 241-AP TANK EARM CALIBRATION PR

FENWAL SERIES 543 THERMOCOURLE SENSING TEMPERATURE CONTROLLER CHECK

PD750 UNIVERSAL TEMPERATURE METER CALIBRATION

UNITED ELECTRIC TEMPERATURE CONTROLS, TYPES B110, B110A, C110, C110A, AND
$10 / 31 / 97$

$5 / 13 / 98$

$5 / 11 / 98$

$5 / 12 / 98$

$5 / 12 / 98$

$5 / 13 / 98$

RELEASE DATE

STATUS REVISING

REVISING

REVISING

ACTIVE

REVISING

REVISING

REVISING

REVISING

REVISING

ACTIVE

REVISING

ACTIVE

ACTIVE

ACTIVE

ACTIVE

ACTIVE

ACTIVE

ACTIVE

ACTIVE

REVISING

DEVELOPING

REVISING

$5 / 27 / 98$

ACTIVE

REVISING

HNF-3410, Rev. 0 
PROCEDURE NUMBER

6-TCD- 464

6-TCD- 466

$6-\mathrm{TCD}-473$

6-TCD-506

6-TCD-512

6-TF-001

6-TF-004

6 -

6-TF-010

6-TF-011

6-TF-013

6-TF-014

6-TF-018

6-TF-022

6-TF-029

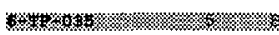

6-TF-037

6-TF-037

6-TF-040

6-TF-0 42

6-TF-044

6-TF-049

6-TF-0.52

\section{RELEASE DATE STATUS}

REVISING

REVISING

REVISING

REVISING

ACT IVE

REVISING

ACTIVE

ACTIVE

ACTIVE

ACTIVE

ACTIVE

ACTIVE

ACTIVE

AĆTIVE

ACT IVE

ACTIVE

ACTIVE

ACTIVE

REVISING

ACTIVE

ACTIVE

ACTIVE

ACTIVE

ACTIVE

HNF-3410, Rev. 0 
$=\dot{=}=x=$

\begin{tabular}{|c|c|c|c|c|c|}
\hline PROCEDURE NUMBER & REV & REV\# & PROCEDURE TITLE & RELEASE DATE & STATUS \\
\hline $6-T F-054$ & 1 & A & CALIBRATION OF "ONE TIME USE" PRESSURE GAUGES & $2 / 26 / 98$ & ACTIVE \\
\hline $6-\mathrm{TF}-055$ & 1 & C & TROUBLESHOOTING \& REPAIR OF LIQUID DETECTOR (LDE \& LLE) & $5 / 18 / 98$ & ACTIVE \\
\hline 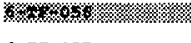 & 40 & 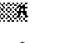 & PM/CAL OF STANDARD DRY WELL MONITORING VANS & $9 / 27 / 96$ & ACTIVE \\
\hline $6-\mathrm{TF}-057$ & 1 & 0 & Dry Well Van Probe Maintenance and Calibration & $8 / 29 / 95$ & ACPIVE \\
\hline $6-\mathrm{TF}-058$ & 2 & G & Verification of FIC Reference Elevation & $1 / 09 / 98$ & ACTIVE \\
\hline $6-T F-059$ & 1 & A & Tennelec Model TC-213 Linear Amplifler and Integral Discriminator Calibra & $1 / 02 / 97$ & ACTIVE \\
\hline soto & 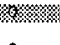 & & 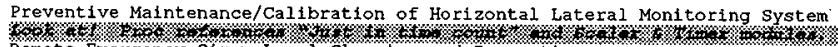 & $2 / 04 / 93$ & ACTIVE \\
\hline $6-\mathrm{TF}-062$ & 0 & 0 & Remote Emergency Siren Local Cleaning and Inspection & $3 / 11 / 93$ & ACTIVE \\
\hline $6-T F-063$ & 0 & 0 & Honda ES-6500 Generator PM & $4 / 02 / 93$ & ACTIVE \\
\hline $6-T F-064$ & 1 & G & Joy WGOL and WGAPOL Oil Free Air Compressor Annual Maintenance & $3 / 19 / 97$ & ACTIVE \\
\hline $6-T F-065$ & 3 & $\mathrm{C}$ & AIR COMPRESSOR INSPECTION & $6 / 05 / 97$ & ACTIVE \\
\hline $6-T F-066$ & 0 & C & Dry Well Van Cable Reheading & $9 / 30 / 96$ & ACTIVE \\
\hline $6-T E-068$ & 0 & 0 & AW270-2 Calibration Procedure for Pressure Alarm Switch PAS-102-3 & $5 / 17 / 93$ & ACTIVE \\
\hline $6-\mathrm{TF}-071$ & 0 & G & Validation of Multiple-Function Instrument Tree (MIT) Thermocouples with $V$ & $3 / 19 / 98$ & ACTIVE \\
\hline $6-\mathrm{TF}-072$ & 0 & B & Testing of Leak Detectors LDE-MPZ-42-1, and 44-1 in 242-A Retention Basin & $1 / 02 / 97$ & ACTIVE \\
\hline $6-T F-076$ & 3 & C & BREATHER FILTER REMOVAL \& REPLACEMENT & $9 / 25 / 97$ & ACTIVE \\
\hline $6-\mathrm{TF}-077$ & 1 & $\mathbf{F}$ & Stack Sampling and Monitoring System Maintenance & $7 / 23 / 98$ & ACTIVE \\
\hline $6-T F-084$ & 1. & A & Ingersoll-Rand Type-30 Oil-Free Air Compressor Inspection & $6 / 13 / 94$ & ACTIVE \\
\hline $6-\mathrm{TF}-085$ & 1 & B & Ingersoll-Rand Type-30 oil Free Air Compressor Maintenance & $1 / 10 / 97$ & ACTIVE \\
\hline $6-\mathrm{TF}-089$ & 0 & B & Inspection of Exit Lights for WTF & $2 / 27 / 98$ & ACTIVE \\
\hline $6-\mathrm{TF}-094$ & 1 & $\mathbf{E}$ & Regenerative Aix Dryer Inspection and Maintenance & $1 / 10 / 97$ & ACTIVE \\
\hline $6-\mathrm{TF}-105$ & 0 & A & Field Calibration of Stevens Total Flowmeter, Model 61R & $2 / 27 / 98$ & ACTIVE \\
\hline $6-T F-111$ & 0 & C & $\begin{array}{l}\text { Matntenance and/or Replacement of FTIR optical Bench and Sample Cell for } 2 \\
\text { W }\end{array}$ & $3 / 02 / 98$ & $\begin{array}{l}\text { ACT IVE } \\
\text { PQY }\end{array}$ \\
\hline $6-\mathrm{TE}-112$ & 0 & A & Maintenance and/or Replacement of Gas Chromatograph $(\mathrm{GC}-31)$ for $241-\mathrm{SY}-101$ & $3 / 02798$ & ACTIVE \\
\hline
\end{tabular}

HNF-3410, Rev. 0 
APPENDIX AB, 241-A-702 AY/AZ TANK EXHAUSTER STACK 296-A-17 AIR FLOW TEST D 1/06/98

APPENDIX AC, 241-A-702 AY/AZ BACKUP TANK AIR ELOW TEST DATA SHEETS

ARPENDIX AD, 241-AN ANNULUS EXHAUSTER STACK 296-A-30 AIR FLOW TEST DATA SH

APPENDIX AE, 241-AP ANNULUS EXHAUSTER STACK 296-A-41 ATR FLOW TEST DATA SH

APPENDIX AF, 241-AW-ANNULUS EXHAUSTER STACK 296-A-28 AIR FLOW TEST DATA SH

APPENDIX AG, 241-AY 101 ANNULUS EXHAUSTER STACK 296-A-18 AIR FLOW TEST DAT

APPENDIX AH, 241-AY 102 ANNULUS EXHAUSTER STACK 296-A-19 AIR FLOW TEST DAT

APPENDIX AI, 241-AZ ANNULUS EXHAUSTER STACK 296-A-20 AIR FLOW TEST DATA SH

APPENDIX AJ, 241-C-105/106 TANK EXHAUSTER STACK 296-P-16 AIR FLOW TEST DAT

APRENDIX AK, 241-SX TANK EXHAUSTER STACK 296-S-15 AIR FLOW TEST DATA SHEET

APPENDIX AM, 242-S HOT CELL EXHAUST STACK 296-S-18 AIR FLOW TEST DATA SHEE

APPENDIX AN, 242-T BUILDING EXHAUST STACK 296-T-17 AIR FLOW TEST DATA SHEE

APPENDIX AO, 244-AR CANYON EXHAUSTER STACK 296-A-13 AIR FLOW TEST DATA SHE

APPENDIX Ä, 244-CR VAULT EXHAUSTER STACK 296-C-5 AIR FLOW TEST DATA SHEET

APPENDIX AQ, 209-E CELL \& BUILDING EXHAUST STACK 296-P-31 AIR FLOW TEST DA APPENDIX AW, 213-W COMPACTOR BUILDING EXHAUST STACK 296-W-3 AIR FLOW TEST

\section{$1 / 06 / 98$}

$4 / 27 / 98$

$4 / 27 / 98$

$4 / 27 / 98$

$4 / 14 / 98$

$7 / 22 / 98$

$1 / 06 / 98$

$2 / 24 / 98$

$12 / 23 / 97$

$12 / 23 / 97$

$12 / 23 / 97$

$1 / 06 / 98$

ACTIVE

ACTrYE

ACrIVE

ACTIVE

ACTIVE

ACTIVE

ACTIVE

ACTIVE

ACTIVE

ACTIVE

ACTIVE

ACTIVE

ACTIVE

ACTIVE

ACTIVE

ACTIVE

ACTIVE

ACTIVE

ACTIVE

$$
H N F-3410 \text {, Rev. } 0
$$




\begin{tabular}{|c|c|}
\hline PROCEDURE NUMBER & REV \\
\hline $6-T F-155 B$ & 1 \\
\hline $6-T F-155 C$ & 1 \\
\hline $6-T F-155 D$ & 1 \\
\hline $6-T F-155 E$ & 1 \\
\hline $6-T F-155 F$ & 1 \\
\hline $6-T F-155 \mathrm{H}$ & 1 \\
\hline $6-T F-155 I$ & 1 \\
\hline $6-T F-155 J$ & 1 \\
\hline $6-T E-155 K$ & 1 \\
\hline $6-T E-155 t$ & 1 \\
\hline $6-T F-155 M$ & 1 \\
\hline $6-\mathrm{TE}-155 \mathrm{~N}$ & A \\
\hline $6-T E-1550$ & A \\
\hline $6-T F-155 P$ & 1 \\
\hline $6-T F-1550$ & $\mathbf{1}$ \\
\hline $6-T E-155 S$ & 0 \\
\hline $6-T E-155 T$ & 1 \\
\hline $6-T F-155 U$ & $A$ \\
\hline $6-T E-156$ & 0 \\
\hline $6-T E-156 \mathrm{AA}$ & 0 \\
\hline $6-\mathrm{TE}-156 \mathrm{AB}$ & 0 \\
\hline $6-T F-156 A C$ & 0 \\
\hline $6-T F-156 \mathrm{AD}$ & 0 \\
\hline $6-T E-156 \mathrm{AE}$ & 0 \\
\hline
\end{tabular}

\section{REV PROCEDURE TITLE}

APPENDIX B, 241-AP TANK EXHAUSTER STACK 296-A-40 AIR FLOW TEST DATA SHEETS

AFPENDIX C, 241-AW TANK EXHAUSTER STACK 296-A-27 AIR FLOW TEST DATA SHEETS

APPENDIX D, 241-SY ANNULUS EXHAUSTER STACK 296-A-22 AIR FLOW TEST DATA SHE

APPENDIX E, 241-SY TANK EXHAUSTER STACK 296-P-23 AIR FLOW TEST DATA SHEETS

APPENDIX F, 241-SY BACKUP EXHAUSTER STACK 296-P-28 AIR FLOW TEST DATA SHEE

APPENDIX H, 244-A DCRT EXHAUSTER STACK 296-A-25 AIR FLOW TEST DATA SHEETS

APPENDIX I, 244-BX DCRT EXHAUSTER STACK 296-B-28 AIR FLOW TEST DATA SHEETS

APPENDIX J, 244-S DCRT EXHAUSTERS STACK 296-S-22 AIR FLOW TEST DATA SHEETS

APPENDIX K, 244-TX DCRT EXHAUSTER STACK 296-T-18 AIR FLOW TEST DATA SHEETS

APPENDIX L, 244-U DCRT EXHAUSTER STACK 296-U-11 AIR FLOW TEST DATA SHEETS

APPENDIX M, 241-SY TANK EXHAUSTER STACK 296-S-25 AIR FLOW TEST DATA SHEETS

APPENDIX N, 241-AZ-702 BUILDING EXHAUST STACK 296-A-43 AIR FLOW TEST DATA

APPENDIX 0, 241-AY/AZ TANK EXHAUST STACK 296-A-42 AIR FLOW TEST DATA SHEET

APPENDIX P, 241-C 106 TANK EXHAUSTER STACK 296-C-6 AIR FLOW TEST DATA SHEE

APPENDIX Q, 500 CFM PORTABLE EXHAUSTER 296-P-41 AIR FLOW TEST DATA SHEETS

APPENDIX S, CORE SAMPLING EXHAUSTER 296-P-33 AIR FLOW TEST DATA SHEET

APPENDIX T, CORE SAMPLING EXHAUSTER 296-P-34 AIR FLOW TEST DATA SHEET

APPENDIX U, 1000 CFM PORTABLE EXHAUSTERS POR-007-SKID E \& POR-008-SKID F A

241-AN K1-5-1 TANK EXHAUSTER 296-R-29 HEPA FILTER AEROSOL

APPENDIX AA, 241-AN TANK FARM ANNULUS INLET FILTER AEROSOL TEST DATA SHEET

APPENDIX AB, 241-AN EF-103 ANNULUS EXHAUSTER 296-A-30 HEPA FILTER AEROSOL

APPENDIX AC, 241-AN EF-104 ANNULUS EXHAUSTER 296-A-30 HEPA FILTER AEROSOL

APPENDIX AD, 241-AP TANK EARM ANNULUS YNLET FILTER AEROSOL TEST DATA SHEET

APPENDIX AE, 241-AP K2-5-1 ANNULUS EXHAUSTER HEPA FILTER AEROSOL TEST DATA
RELEASE DATE

$2 / 23 / 98$

$4 / 27 / 98$

$12 / 23 / 97$

$12 / 23 / 97$

$1 / 05 / 98$

$1 / 06 / 98$

$1 / 05 / 98$

$12 / 29 / 97$

$12 / 30 / 97$

$1 / 05 / 98$

$12 / 30 / 97$

$1 / 07 / 98$

$1 / 20 / 98$

$5 / 18 / 98$

$1 / 07 / 98$

$7 / 17 / 98$

1/05/98

$7 / 09 / 98$

10/02/97

$6 / 12 / 97$

2/23/98

$6 / 12 / 97$

$3 / 04 / 98$

$12 / 04 / 97$
STATUS

\section{ACTIVE}

ACTIVE

ACTIVE

ACTIVE

ACTIVE

ACTIVE

ACTIVE

ACTIVE

ACTIVE

ACTIVE

ACTIVE

ACTIVE

ACTIVE

ACTIVE

ACTIVE

ACTIVE

ACTIVE

ACTIVE

ACTIVE

ACTIVE

ACTIVE

ACTIVE

ACTIVE

ACTIVE

$$
\text { HNF-34.10, Rev. } 0
$$




\begin{tabular}{|c|c|c|c|c|c|}
\hline PROCEDURE NUMBER & REV & REV\# & RROCEDURE TITLE & RELEASE DATE & STATUS \\
\hline $6-T F-156 \mathrm{AF}$ & 0 & $\mathbf{E}$ & APPENDIX AE, 241-AP K2-5-2 ANNULUS EXHAUSTER HEPA EILTER AEROSOL TEST DATA & $3 / 06 / 98$ & ACTIVE \\
\hline $6 \sim T F-156 A G$ & 0 & A & APPENDIX AG, 241-AW TANK FARM ANNULUS INLET FILTER AEROSOL TEST DATA SHEET & $4 / 27 / 98$ & ACTIVE \\
\hline $6-T F-156 A I$ & 0 & A & APPENDIX AI, 241-AW K2-5-2. ANNULUS EXHAUSTER 296-A-28 HEPA FILTER AEROSOL & $4 / 27 / 98$ & ACTIVE \\
\hline 6-TF-156AJ & 0 & B & APPENDIX AJ, 241-AY 101 ANNULUS EXHAUSTER 296-A-18 HEPA FILTER AEROSOL TES & $3 / 06 / 98$ & ACTIVE \\
\hline $6-T F-156 A K$ & 0 & D & A.PPENDIX AK, 241-AY 102 ANNULUS EXHAUSTER 296-A-19 HERA FILTER AREOSOL TES & $2 / 23 / 98$ & ACTIVE \\
\hline $6-T F-156 A L$ & 0 & A & APPENDIX AL, 241-AZ ANNULUS EXHAUSTER 296-A-20 HEPA FILTER AEROSOL TEST DA & $7 / 31 / 95$ & ACTIVE \\
\hline $6-T F-156 A M$ & 0 & B & APPENDIX AM, 241-SY TANK FARM ANNULUS INLET EILTER AEROSOL TEST DATA SHEET & $5 / 30 / 97$ & ACTIVE \\
\hline 6-TF-156AN & 0 & 0 & APPENDIX AN, 241-SY ANNULUS EXHAUSTER 296-P-22 HEPA FILTER AEROSOL TEST & $3 / 30 / 95$ & ACTIVE \\
\hline $6-T F-156 B$ & A & 0 & A.RPENDIX B, 1000 CEM PORTABLE EXHAUSTERS POR-007-SKID E \& POR-008-SKID F H & $7 / 09 / 98$ & ACTIVE \\
\hline $6-\mathrm{TF}-156 \mathrm{DA}$ & 0 & B & APPENDIX DA, 244-A DCRT INLET FILTER AEROSOL TEST DATA SHEETS & $10 / 14 / 97$ & ACTIVE \\
\hline $6-\mathrm{TF}-156 \mathrm{DB}$ & 0 & B & APPENDIX DB, 244-A DCRT EXHAUSTER 296-A-25 HEPA FILTER AEROSOL TEST & $10 / 14 / 97$ & ACTIVE \\
\hline $6-T F-156 D D$ & 0 & A & APPENDIX DD, 244-BX DCRT EXHAUSTER 296-B-28 HEPA FILTER AEROSOL TEST DATA & $6 / 23 / 97$ & ACTIVE \\
\hline $6-T F-156 \mathrm{DE}$ & 0 & A & APPENDIX DE, 244-S DCRT INLET FILTER AEROSOL TEST DATA SHEETS & $10 / 16 / 97$ & ACTIVE \\
\hline $6-\mathrm{TF}-156 \mathrm{DE}$ & 0 & B & APPENDIX DE, 244-S DCRT EXHAUSTER 296-S-22 HERA FILTER AEROSOL TEST DATA $S$ & $10 / 27 / 97$ & ACTIVE \\
\hline $6-\mathrm{TF}-156 \mathrm{DG}$ & 0 & $\mathrm{~B}$ & APPENDIX DG, 244-TX DCRT INLET FILTER AEROSOL TEST DATA SHEETS & $10 / 16 / 97$ & ACTIVE \\
\hline $6-\mathrm{TF}-156 \mathrm{DH}$ & 0 & $\mathrm{~B}$ & APPENDIX DH, 244-TX DCRT EXHAUSTER 296-T-18 HEPA FILTER AEROSOL TEST DATA & $9 / 26 / 97$ & ACTIVE \\
\hline $6-\mathrm{TF}-156 \mathrm{DI}$ & 0 & 0 & ARPENDIX DI, 244-U DCRT INLET FILTER AEROSOL TEST DATA SHEETS & $8 / 31 / 95$ & ACTIVE \\
\hline $6-\mathrm{TF}-156 \mathrm{DJ}$ & 0 & 0 & ARPENDIX DJ, 244-U DCRT EXHAUSTER 296-U-11 HEPA FILTER AREOSOL TEST DATA $S$ & $8 / 31 / 95$ & ACTIVE \\
\hline $6-\mathrm{TF}-156 \mathrm{FA}$ & 0 & A & APPENDIX FA, 204-AR LOAD OUT ROOM EXHAUSTER 296-A-26 HEPA FILTER AEROSOI T & $6 / 23 / 97$ & ACTIVE \\
\hline $6-\mathrm{TF}-156 \mathrm{FB}$ & 0 & A & APPENDIX FB, 204-AR CATCH TANK EXHAUSTER 296-A-26 HERA FILTER AEROSOL TEST. & $6 / 23 / 97$ & ACTIVE \\
\hline $6-T F-156 F C$ & 0 & B & APPENDIX FC, 209-E CELL \& BUILDING EXHAUSTER 296-P-31 HEPA FILTER AEROSOL & $12 / 23 / 97$ & ACTIVE \\
\hline $6-\mathrm{TF}-156 \mathrm{FH}$ & 0 & C & APPENDIX FH, 242-S HOT CELL EXHAUSTER 296-S-18 HEPA FILTER AEROSOL TEST DA & $6 / 12 / 97$ & ACTIVE \\
\hline
\end{tabular}

HNF-3410, Rev. 0 


\section{PROCEDURE NUMBER REV REV\# PROCEDURE TITLE}

\section{6-TF-156FI}

6-TF-156FK

6-TF-156FL

$6-\mathrm{TF}-156 \mathrm{FN}$

$6-\mathrm{TF}-156 \mathrm{FO}$

6-TF-156FW

6-TF-156PB

6-TF-156PC

6-TE-156TB

6-TF-156TC

6-TF-156TD

6-TF-156TE

6-TF-156TF

6-TF-156TG

6-TF-156TH

6-TF-156TI

6-TF-156TJ

6-TF-156TK

6-TF-156TL

6-TF-156TM

6-TF-156TN

6-TF-156TO

6-TF-156TP

6-TF-156TQ
APPENDIX FI, 242-T BUILDING EXHAUSTER 296-T-17 HEPA FILTER AEROSOL TEST DA

APPENDIX FK, 244-CR VAULT EXHAUSTER 296-C-5 HEPA EILTER AEROSOL TEST DATA

ARPENDIX FL, 244-CR VAULT EXHAUSTER 296-C-5 TOP OVERALL HERA FILTER AEROSO APPENDIX FN, 242-AZ-702 VENTCELL INLET FILTER AEROSOL TEST DATA SHEETS

APPENDIX FO, 241-AZ 702 BUILDING EXHAUSTER 296-A-43 AEROSOL TEST DATA SHEE ARPENDIX FW, 213-W COMPACTOR BUILDING EXHAUSTER 296-W-3 HEPA. FILTER AEROSO APPENDIX PB, CORE SAMPLING EXHAUSTER 296 - P-33 HEPA FILTER AEROSOL TEST DA APPENDIX PC, CORE SAMPLING EXHAUSTER 296-P-34 HERA FILTER AEROSOL TEST DAT ARPENDIX TB, 241-A-702 AY/A2 TANK EXHAUSTER 296-A-17 HEPA FILTER AEROSOL T APPENDIX TC, 241-A $\rightarrow 702$ AY/AZ BACKUP EXHAUSTER 296-P-26 HEPA FILTER AEROSOL APPENDIX TD, 241-AN EF-101 TANK EXHAUSTER 296-A-29 HEPA FILTER AEROSOL TES AEPENDIX TE, 241-AN EF-102 TANK EXHAUSTER 296-A-29 HEPA EILTER AEROSOL TES APPENDIX TF, 241-AP K1-5-1 TANK EXHAUSTER 296-A-40 HEPA FILTER AEROSOL TES APPENDIX TG, 241-AP K1-5-2 TANK EXHAUSTER 296-A-40 HEPA FILTER AEROSOL TES ARPENDIX TH, 241-AW K1-5-1 TANK EXHAUSTER 296-A-27 HEPA FILTER AEROSOL TES ARPENDIX TI, 241-AW K1-5-2 TANK EXHAUSTER 296-A-27 HEPA FILTER AEROSOL TES APPENDIX TJ, 241-C 105/106 TANK INLET FILTER AEROSOL TEST DATA SHEETS ARPENDIX TK, 241-C 105/106 TANK EXHAUSTER 296-P-16 HEPA EILTER AEROSOL TES APRENDIX TL, 241-SX TANK INLET EILTER AEROSOL TEST DATA SHEETS

APPENDIX TM, 241-SX TANK EXHAUSTER 296-S-15 HERA EILTER AREOSOL TEST APPENDIX TN, 241-SY TANK INLET FILTER AEROSOL TEST DATA SHEETS ARPENDIX TO, 241-SY TANK EXHAUSTER 296-P-23 HEPA FILTER AEROSOL TEST APPENDIX TP, 241-SY BACKUP TANK EXHAUSTER 296-P-28 HEPA FILTER AEROSOL TES APPENDIX TQ, 241-SY TANK EXHAUSTER 296-S-25 HEPA FILTER AEROSOL TEST DATA
RELEASE DATE $6 / 24 / 97$

$6 / 24 / 97$

$7 / 08 / 97$

$2 / 25 / 98$

$10 / 16 / 97$

$6 / 12 / 97$

$7 / 17 / 98$

$1 / 05 / 98$

$6 / 24 / 97$

$1 / 18 / 95$

$2 / 23 / 98$

$2 / 23 / 98$

$11 / 25 / 97$

$3 / 04 / 98$

$5 / 08 / 98$

$5 / 08 / 98$

$5 / 14 / 98$

$5 / 30 / 97$

$4 / 18 / 97$

$7 / 01 / 97$

$6 / 03 / 97$

$1 / 19 / 95$

$10 / 02 / 95$

$10 / 02 / 95$
STATUS ACTIVE

ACTIVE

ACTIVE

ACTIVE

ACTIVE

ACTIVE

ACTIVE

ACTIVE

ACTIVE

ACTIVE

ACTIVE

ACTIVE

ACTIVE

ACTIVE

ACTIVE

ACTIVE

ACTIVE

ACTIVE

ACTIVE

ACTIVE

ACTIVE

ACTIVE

ACTIVE

ACrive

HNF-3410, Rev. 0 


\begin{tabular}{|c|c|}
\hline PROCEDURE NUMBER & REV \\
\hline $6-T F-156 \mathrm{TR}$ & 0 \\
\hline $6-T F-156 \mathrm{TS}$ & A \\
\hline $6-\mathrm{TE}-156 \mathrm{TT}$ & 0 \\
\hline $6-\mathrm{TF}-156 \mathrm{TT}$ & 0 \\
\hline $6-\mathrm{TF}-156 \mathrm{TU}$ & A \\
\hline $6-\mathrm{TF}-156 \mathrm{TV}$ & 0 \\
\hline 6-TF-156TW & 0 \\
\hline $6-T F-157$ & 0 \\
\hline $6-T F-157 A$ & 0 \\
\hline $6-T F-157 A X$ & 0 \\
\hline $6-T F-157 \mathrm{~B}$ & 0 \\
\hline $6-\mathrm{TF}-157 \mathrm{BX}$ & 0 \\
\hline $6-T F-157 B Y$ & 0 \\
\hline $6-T F-157 C$ & 0 \\
\hline 6-TF-157DU & 0 \\
\hline $6-T F-157 \mathrm{EW}$ & 0 \\
\hline $6-\mathrm{TF}-157 \mathrm{QB}$ & 0 \\
\hline $6-T F-157 Q C$ & 0 \\
\hline $6-T F-157 Q T$ & 0 \\
\hline $6-T F-157 S$ & 0 \\
\hline $6-T F-157 S S$ & 0 \\
\hline $6-T F-157 \mathrm{SX}$ & 0 \\
\hline $6-T F-157 \mathrm{~T}$ & 0 \\
\hline 6-TF-157TX & \\
\hline
\end{tabular}

PROCEDURE TITLE

APPENDIX TR, 241 AY/AZ-702 TANK INLET FILTER AEROSOL TEST DATA SHEETS

APPENDIX TS, 241 AY/AZ TANK INLET EXHAUSTER 296-A-42 AEROSOL TEST DATA SHE

ARPENDIX TT, 241-C 106 TANK EXHAUSTER 296-C-6 AEROSOL TEST DATA SHEETS

APPENDIX TT, 241-C 106 TANK EXHAUSTER 296-C-6 AEROSOL TEST DATA SHEETS

APPENDIX TU, 500 CFM PORTABLE EXHAUSTER 296-P-41 HEPA FILTER AEROSOL TEST

APPENDIX TV, 241-AN TANK INLET FILTER AEROSOL TEST DATA SHEETS

ARPENDIX TW, 241-AW TANK INLET FILTER AEROSOL TEST DATA SHEET

241-QC TANK FARM BREATHER FILTER AEROSOL TEST DATA SHEETS

APPENDIX A, 241-A TANK FARM BREATHER FILTER AEROSOL TEST DATA SHEETS

APPENDIX AX, 241-AX TANK FARM BREATHER FILTER AEROSOL TEST DATA SHEETS

APPENDIX B, 241-B TANK FARM BREATHER AEROSOL TEST DATA SHEETS

APPENDIX BX, 241-BX TANK FARM BREATHER FILTER AEROSOL TEST DATA SHEETS

APPENDIX BY, 241-BY TANK FARM BREATHER FILTER AEROSOL TEST DATA SHEETS

APPENDIX $C$, 241-C TANK FARM BREATHER FILTER AEROSOL TEST DATA SHEETS

APPENDIX DU, 244-U DCRT BREATHER FILTER AEROSOL TEST DATA SHEETS

APPENDIX EW, 241-EW-151 CATCH TANK BREATHER FILTER AEROSOL TEST DATA SHEET

APPENDIX QB, 241-BX-101 BREATHER FILTER AEROSOL TEST DATA SHEET

AEPENDIX QC, 241-C 103 BREATHER FILTER AEROSOL TEST DATA SHEETS

APPENDIX QT, 241-TX-113 BREATHER FILTER AEROSOL TEST DATA SHEETS

APPENDTX 5 , 241-S TANK FARM BREATHER FILTER AEROSOL TEST DATA SHEETS

APFENDIX SS, 241-S-304 CATCH TANK BREATHER FILTER AEROSOL TEST DATA SHEETS

APPENDIX SX, 241-SX TANK FARM BREATHER FILTER AEROSOL TEST DATA SHEETS

APPENDIX T, 241-T TANK FARM BREATHER EILTER AEROSOL TEST DATA SHEETS

APPENDIX TX, 241-TX TANK FARM BREATHER FILTER AEROSOL TEST DATA SHEETS
RELEASE DATE

$1 0 \longdiv { 2 6 / 9 7 }$

$10 / 27 / 97$

$8 / 20 / 96$

$5 / 28 / 98$

$7 / 30 / 97$

$7 / 14 / 98$

$5 / 14 / 98$

10/23/97

$2 / 23 / 98$

10/28/97

$10 / 27 / 97$

$10 / 27 / 97$

$10 / 23 / 97$

$2 / 25 / 98$

$1 / 04 / 96$

$5 / 30 / 97$

$9 / 26 / 97$

$12 / 22 / 97$

$10 / 20 / 97$

$10 / 16 / 97$

$6 / 30 / 97$

$10 / 23 / 97$

10/20/97

$1 / 13 / 98$
STATUS

ACTIVE

ACTIVE

ACTIVE

ACTIVE

ACTIVE

ACTIVE

ACTIVE

ACTIVE

ACTIVE

ACTIVE

ACTIVE

ACTIVE,

ACTIVE

ACTIVE

ACT IVE

ACTIVE

ACTIVE

ACTIVE

ACTIVE

ACTIVE

ACTIVE

ACTIVE

ACTIVE

ACTIVE

HNF-3470, Rev. 0 


\begin{tabular}{|c|c|c|c|c|c|}
\hline PROCEDURE NUMBER & REV & REV\# & PROCEDURE TITLE & RELEASE DATE & STATUS \\
\hline $6-\mathrm{TF}-157 \mathrm{TY}$ & 0 & B & 241-TY TANK FARM BREATHER FILTER AEROSOL TEST DATA SHEETS & $9 / 30 / 97$ & ACTIVE \\
\hline $6-T F-157 U$ & 0 & B & APPENDIX U, 241-U TANK FARM BREATHER FILTER AEROSOL TEST DRTA SHEETS & $9 / 30 / 97$ & ACTIVE \\
\hline $6-T F-157 V A$ & A & 0 & APPENDIX VA, 6241-A CROSS SITE FACILITY BREATHER FILTER AEROSOL TEST DATA & $3 / 06 / 98$ & ACTIVE \\
\hline $6-\mathrm{TF}-157 \mathrm{VV}$ & A & 0 & APPENDIX VV, 6241-V CROSS SITE FACILITY BREATHER FILTER AEROSOL TEST DATA & $3 / 06 / 98$ & ACTIVE \\
\hline $6-\mathrm{TF}-157 \mathrm{~W}$ & 0 & A & APPENDIX $w, 213-w$ RETENTION TANK BREATHER FILTER AEROSOL TEST DATA SHEETS & $12 / 07 / 95$ & ACTIVE \\
\hline $6-T F-158$ & 0 & D & 241-SY WASTE TANK AIR FLOW MEASUREMENT DATA SHEETS & $10 / 23 / 97$ & ACTIVE \\
\hline $6-T F-158 A N$ & 0 & c & APPENDIX AN, 241-AN WASTE TANK AIR FLOW MEASUREMENT DATA SHEETS & $4 / 22 / 97$ & RCTIVE \\
\hline $6-\mathrm{TF}-1.58 \mathrm{C}$ & 0 & c & APPENDIX C, 241-C 105/106 WASTE TANK AIR FLOW MEASUREMENT DATA SHEETS & $4 / 22 / 97$ & ACTIVE \\
\hline $6-T F-158 S X$ & 0 & $B$ & ARPENDIX SX, 241-SX WASTE TANK AIR ELOW MEASUREMENT DATA SHEETS & $4 / 15 / 97$ & ACTIVE \\
\hline $6-T F-158 S X$ & B & 0 & APPENDIX SX, 241-SX WASTE TANK AIR ELOW MEASUREMENT DATA SHEETS & & REVISING \\
\hline $6-\mathrm{TF}-158 \mathrm{~S} X$ & 0 & $\mathrm{~B}$ & APPENDIX SY, 241-SY WASTE TANK AIR ELOW MEASUREMENT DATA SHEETS & $4 / 22 / 97$ & ACTIVE \\
\hline $6-T F-160$ & 1 & $\mathbf{E}$ & Static Bonding for Portable Equipment & $11 / 13 / 97$ & ACTIVE \\
\hline (20) & 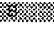 & 器 & $\begin{array}{l}\text { EBERLINE AMS-4 CONTINUOUS AIR MONITOR CALIBRATION } \\
\text { H }\end{array}$ & $10 / 31 / 97$ & ACTIVE \\
\hline 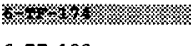 & 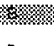 & 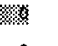 & 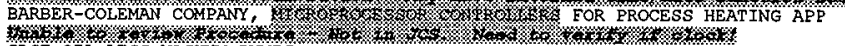 & & REVISING \\
\hline $6-T \mathrm{~F}-182$ & A & 0 & GAST OIL-LESS VACUUM PUMPS & & DEVELOPING \\
\hline $6-T F-183$ & 0 & 0 & 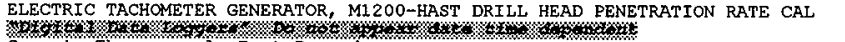 & $5 / 03 / 95$ & ACTIVE \\
\hline $6-\mathrm{PF}-191$ & 0 & C & Generic Thermocouple Test Procedure & $6 / 05 / 98$ & ACTIVE \\
\hline $6-\mathrm{TF}-192$ & 0 & 0 & General Electric Clrcuit Breaker Type AK-50/75/100 & $8 / 26 / 94$ & ACTIVE \\
\hline $6-\mathrm{TF}-197$ & 0 & A & 2704-HV EMERGENCY LIGHTS INSPECTION & $1 / 30 / 97$ & ACTIVE \\
\hline $6-T F-198$ & 0 & 0 & DRYWELL VAN PROBE CONNECTORS & & DEVELOPING \\
\hline $6-\mathrm{TF}-200$ & 0 & 0 & MOORE INDUSTRIES MODEL ALX DIRECT CURRENT & $10 / 26 / 95$ & ACTIVE \\
\hline 6-TF-201 & 0 & 0 & 244-AR VAULT CRANE, ELECT & & DEVELOPING \\
\hline $6-T F-202$ & 0 & 0 & 1000 CEM PORTABLE EXHAUSTER (296-P-28) PREVENTIVE MAINTENANCE \& FUNCTIONAL & & DEVELOPING \\
\hline $6-\mathrm{TF}-203$ & 0 & 0 & 4/20ma Repeater Power Supply Check & $10 / 26 / 95$ & ACPIVE \\
\hline
\end{tabular}

HNF-3410, Rev. 0 
PROCEDURE NUMBER

6-TF-204

$6+\mathrm{TF}-205$

6-TF-206

$6-\mathrm{TF}-208$

6-TF-209

$6-\mathrm{TF}-210$

6-TF-212

6-TF-213

6-TF-214

6-TE-215

6-TF-216

6-TF-219

6-TF-220

6-TF-221

6-TF-225

6-TF-227

6-TE-228

6-TF-233

6-TF-237

6-TF-239

6-TF-240

6-TF-241

6-TF-242

6-TF-243

\section{REV\# PROCEDURE TITLE}

INSPECTION OF EMERGENCY LIGHTS TANK EARMS

MERCOID PRESSURE CONTROL SWTTCH CALIBRATION INSTRUCTIONS

DRY WELL VAN AUX GENERATORS

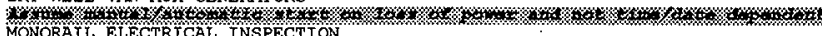

CASS POWERBLOC POWER CONDITIONING, $2750 \mathrm{E}$

FUNCTIONAL TEST OF CASS ALARMS EOR POWER CONDITIONING SYSTEM - 2750E BUTLD

INSP AND MAINT OF 252-S SWITCHGEAR BATTERIES (TYPE H34P)

Onan Diesel Generator

GM Delco Diesel Generator $300 \mathrm{KW}$ Inspection \& Operation

Ili-Case Diesel Generator $110 \mathrm{KW}$ Inspection Operation

241-A-701 300kW GM-Delco Standby Diesel Generator Cold Automatic Start, In

ILI-CASE $110 \mathrm{~kW}$ STANDBY DIESEL GENERATOR ATS INSPECTION \& SERVICE

Inspection of Cathodic Protection System Rectifiers

INSPECTION OF EAST TANK FARM TRANSFORMERS

MANUAL TEMPERATURE READINGS FOR WEST AREA TANK FARMS

Baltimore Cooling Tower Maintenance

OPERATING LOAD CHECK \& THERMAL HEATING SURVEY OF THE 241-AP AREA DISTRIBUT

SUPRLY AND EXHAUST FAN INSPECTION

CAL OF ISA-34RAL CARBON MONOXIDE (CO) MONITOR

BELCO INSPECTION

PORTABLE ELECTRIC TOOLS INSPECTION

Digital Panel Meter Calibration Instructions

TRANSMITTER/TRANSDUCER CALIBRATION INSTRUCTIONS Ho.
RELEASE DATE

$8 / 17 / 95$

$7 / 23 / 98$

$10 / 16 / 95$

$3 / 05 / 98$

$3 / 05 / 98$

$7 / 23 / 98$

$10 / 26 / 94$

STATUS

DEVELOPING

ACTIVE

DEVELOPING

DEVELOPING

DEVELORING

DEVELORING

ACTIVE

ACTIVE

ACTIVE

ACTIVE

ACTIVE

ACTIVE

ACTIVE

$5 / 28 / 97$

ACTIVE

DEVELOPING

DEVELOPING

$3 / 19 / 97$

$10 / 29 / 97$

$3 / 28 / 96$

$4 / 05 / 96$

ACTIVE

DEVELOPING

ACTIVE

ACTIVE

ACTIVE

DEVELOPING

$3 / 19 / 97$

ACTIVE

$3 / 19 / 97$

ACTIVE

HNF-3410, Rev. 0 


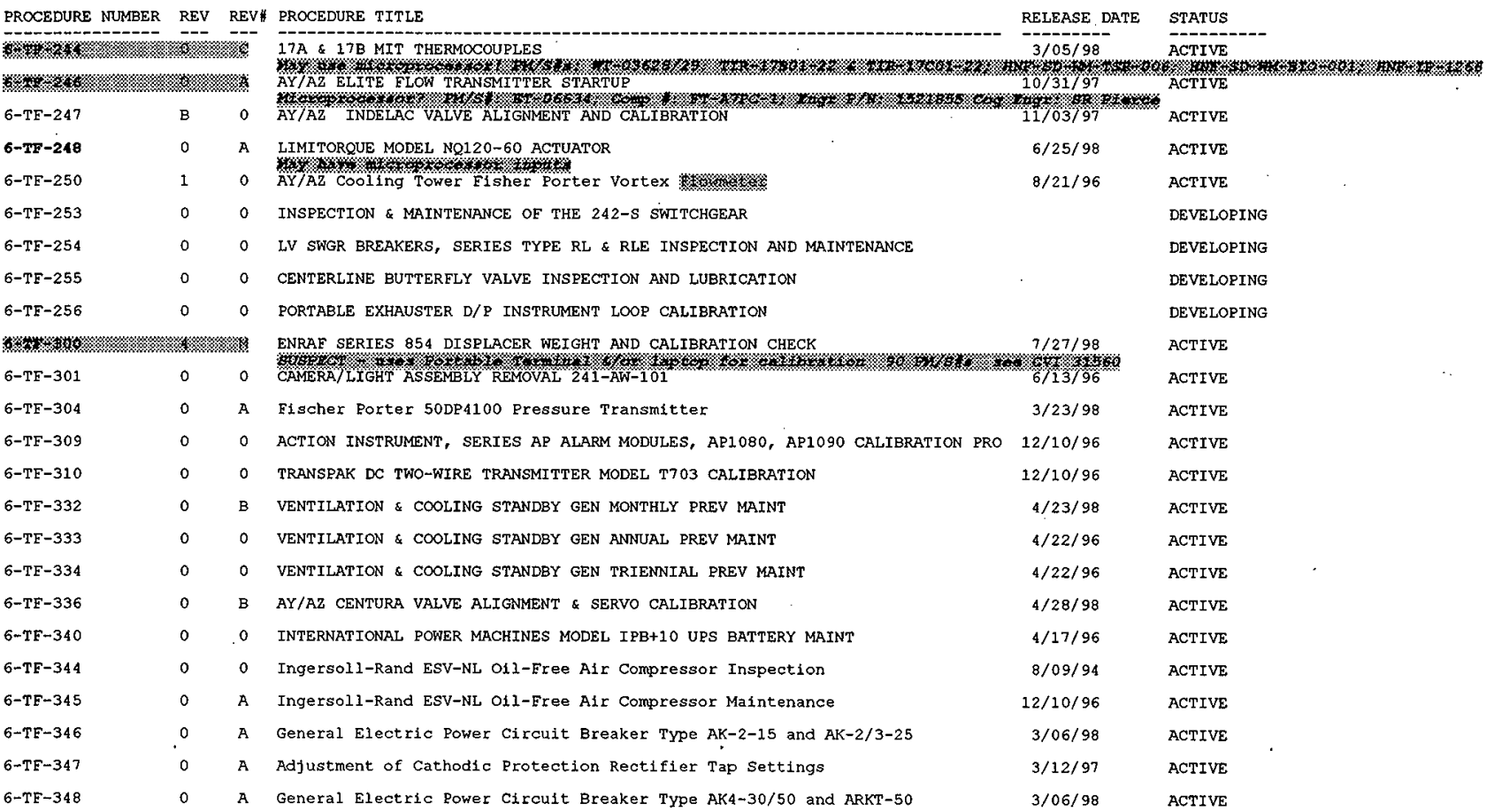

$$
\text { HNF-3410, Rev. } 0
$$


PROCEDURE NUMBER REV REVH PROCEDURE TITLE

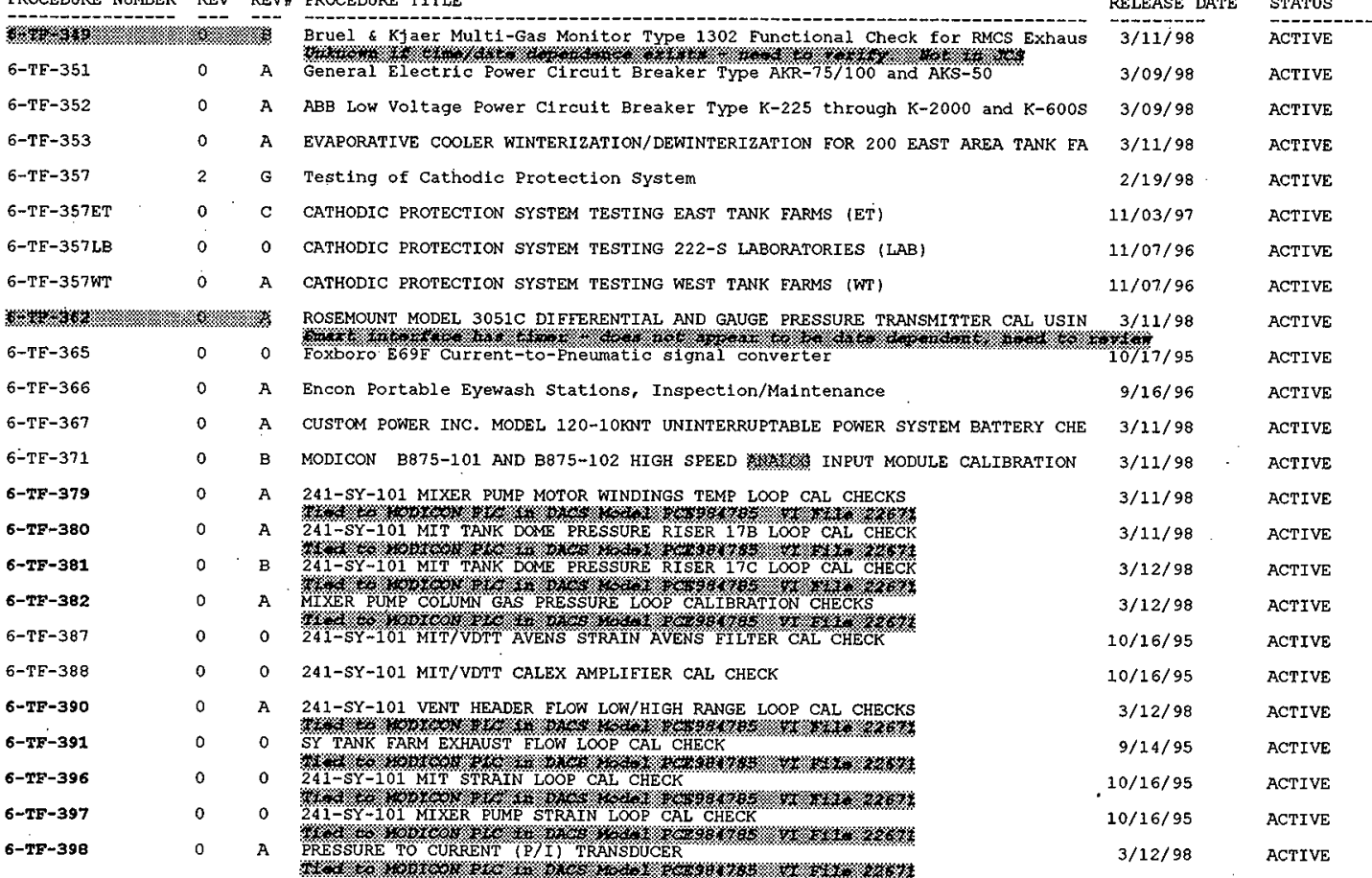

HNF-3410, Rev. 0 


\begin{tabular}{|c|c|c|c|c|c|}
\hline RROCEDURE NUMBER & REV & REV\# & PROCEDURE TITLE & RELEASE DATE & STATUS \\
\hline $6-T P-400$ & 0 & 0 & 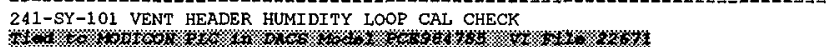 & $10 / 09 / 95$ & ACTIVE \\
\hline 6-TF-401 & 0 & 0 & 241-SY-101 VENT HEADER TEMPERATURE LOOP CAL CHECK & $10 / 09 / 95$ & ACTIVE \\
\hline $6-T R-405$ & 0 & 0 & 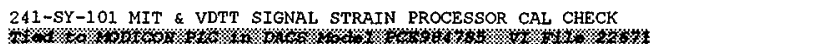 & $10 / 16 / 97$ & ACTIVE \\
\hline $6-77-406$ & 0 & 0 & $241-5 Y-101$ VDTT STRAIN LOOP CAL CHECK & $10 / 16 / 95$ & ACTIVE \\
\hline $6-T F-408$ & 1 & $\cdot P$ & 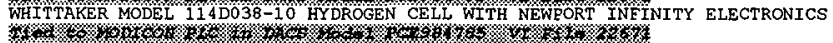 & $6 / 17 / 98$ & ACTIVE \\
\hline $6-T 5-412$ & 0 & 0 & 241-SY-101 TEMP/PRESSURE INDICATING TRANSIMITTER NEWEORT MODEL Q2000 SERIES & $9 / 19 / 95$ & ACTIVE \\
\hline $6-\mathrm{TP}-413$ & 0 & 0 & 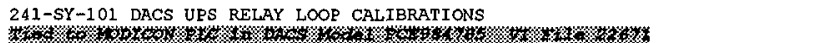 & $10 / 16 / 95$ & ACTIVE \\
\hline $6-T F-414$ & 0 & 0 & $\begin{array}{l}241-S Y-101 \text { THRE CHANNEL RECORDER CHESSELI MODEL } 345 \text { CALIBRATION CHECK } \\
3 \%\end{array}$ & $7 / 26 / 95$ & ACTIVE \\
\hline $6-T E-417$ & 0 & 0 & 241-SY-101 FOXBORO DIFFERENTIAL PRESSURE TRANSMITTERS CALIBRATION CHECK & $7 / 26 / 95$ & ACTIVE \\
\hline $6-T F-423$ & 0 & 0 & 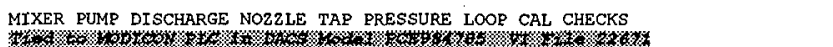 & $9 / 21 / 95$ & ACTIVE \\
\hline 6-TF-424 & 0 & B & MIXER PUMP WOLUTE PRESSORE LOOP CAL CHECKS & $3 / 13 / 98$ & ACTIVE \\
\hline $6-\mathrm{TF}-427$ & 0 & 0 & FENWAL MODEL 54 TEMPERATURE CONTROLLER TC1 \& TC2 & $10 / 26 / 95$ & ACTIVE \\
\hline $6-\mathrm{TE}-429$ & 0 & 0 & ASHCROFT XLDP, ELECTRONIC DIEEERENTIAL PRESSURE CAL & $10 / 26 / 95$ & ACTIVE \\
\hline $6-\mathrm{TF}-431$ & 0 & 0 & DIFFERENTIAL PRESSURE LOOR VERIFICATION & $10 / 26 / 95$ & ACTIVE \\
\hline $6-\mathrm{TF}-432$ & 0 & $\mathbf{B}$ & CAMERA/LIGHT ASSEMBLY INSTALLATION/REMOVAL FOR 241-SY-101 RISER 5A MULTI-P & $4 / 17 / 98$ & ACTIVE \\
\hline $6-\mathrm{TF}-433$ & 0 & o & DIFFERENTIAL TEMPERATURE LOOP VERIFICATION & $10 / 26 / 95$ & RCTIVE \\
\hline $6-T F-434$ & 1 & A & 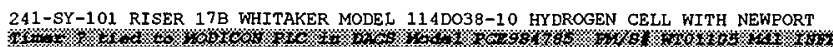 & $3 / 13 / 98$ & ACTIVE \\
\hline $6-T F-435$ & 0 & 0 & 241-SY NITROGEN DISTRIBUTION CABINET LOW PRESSURE ALARM & $12701 / 95$ & ACTIVE \\
\hline $6-T E-436$ & 0 & 0 & High Sheath Temp Switch Calibration & $9 / 21 / 95$ & ACTIVE \\
\hline $6-T E-437$ & 0 & 0 & MIXER PUMP MOTOR WINDINGS INSULATION RESISTANCE CHECKS & $1 / 18 / 96$ & ACTIVE \\
\hline $6-\mathrm{TF}-438$ & 0 & B & removal of gaskets \& valve packing containing asbestos & $3 / 13 / 98$ & ACTIVE \\
\hline $6-\mathrm{TE}-439$ & 0 & 0 & WEST $400 \& 800$ SERIES TEMPERATURE CONTROLLER CALIBRATION INSTRUCTIONS & $9 / 18 / 95$ & ACTIVE \\
\hline
\end{tabular}

HNF-34.10, Rev. 0 
PROCEDURE NUMBER REV REV\# PROCEDURE TITLE (Y)

$6-T F-441$

$6-T F-443$

$6-\mathrm{TF}-444$

5)

6-TF-455

6-TE- 498

6-TF-500

6-TF-501

6-TF-507

6-TF-509

6-T -510

6-TE-511

6-T $5-512$

6-TF-513

6-TF-514

6-TE-515

6-TF-516

$6-T F-516$

$6-\mathrm{TF}-523$

$6-T E-525$

$6-\mathrm{TF}-526$

$6-T F-528$

$6-T F-529$

AIR ROTOMETER hitaker model 114d038-10 hydrogen cell with newport infinity electrontc

MOORE IND. TCA/STD THERMOCOUPLE ALARM \& CONTROLI

PURGING INSTRUMENT DCRT DIP TUBES FOR 244-BX

MIXER PUMP OIL CHANGEOUT

MAC MONITOR BATTERY CHARGER FIELD PROGRAMMING \& MANUAL EQUALIZER INST \% Circuit Breaker Inspection

KOBELCO BREATHING AIR COMPRESSOR PM

ACTION INST SERIES AP ALARM MOD CAL

CAL IRD NECHANALYSIS 5806 AMPLIFIER

Eberline Beta Air Monitor Models AMS-3, AMS-3A, AMS-3A-1 and 700300

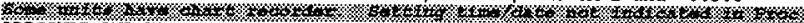
PRESSURE AND VACUUM GAUGES CALIBRATION

DWYER MAGNEHELIC DIFFERENTIAL PRESSURE SERIES 2000 AND CAPSAHELIC DIFFEREN

Thermon Cont ET-HL-1, ET-0600 \& Love Limit cont $48,49 \& 50$

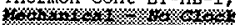

Foxboro Differential Pressure Transmitter Series 13, 13A, $13 \mathrm{H} \& 15$

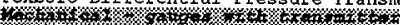

Dwyer Phothelic Serles 3000 \& Capsu-Photohelic series 43000 Diff switch

Wy.

Bajley Model 740 Milivolt Converters

and

Bailey 7000 Divider Model 753

Basez

Bailey 7000 Divider Model 753

BAILEY 7000 VOLTAGE TO EREQUENCY CONVERTER MODEL 741 CALIBRATION EROCEDURE

ACROMAG SERIES 800 PNEUMATIC CONVERTER CALIBRATION PROCEDURE

ROBERTSHAW MODEL 443 AND 445 ELECTRO-PNEUMATIC CONVERTER CALIBRATION PROCE

ACTION MODELS 3300 AMD 7500 CD-TO FREQUENCY CONVERTER CALIBRATION PROCEDUR

ACTION MODEL AP4440 SERIES MATHEMATICAL ROOT EXTRACTOR
RELEASE DATE STATUS

$6 / 16 / 98$

$1 / 29 / 96$

RCTIVE

ACTIVE

$10 / 31 / 97 \quad$ ACTIVE

2/06/96 ACTIVE

$2 / 22 / 96$ B/15/96 \%

4/02/96 ACTIVE

10/01/96 ACTIVE

TRANSFERED

3/16/98 ACTIVE

9/15/97 ACTIVE

3/16/98 ACTIVE

9/15/97 ACTIVE

3/17/98 ACTIVE

6/11/97 ACTIVE

6/11/97 ACTIVE

8/21/96 ACTIVE

8/21/96 ACTIVE

REVISING

REVISING

REVISING

REVISING

REVISING

REVISING

HNF-3410, Rev. 0 


\begin{tabular}{|c|c|c|c|c|c|}
\hline PROCEDURE NUMBER & REV & REV\# & PROCEDURE TITLE & RELEASE DATE & STATUS \\
\hline $6-T F-533$ & A & 2 & 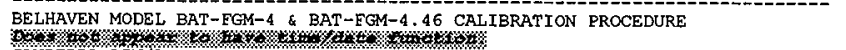 & $1 / 20 / 98$ & ACTIVE \\
\hline $6-T F-543$ & B & 0 & CANBERRA LIN/LOG RATEMETER MODULE 1481L CALIBRATION PROCEDURE & & REVISING \\
\hline $6-T F-549$ & B & 0 & HANFORD-BUILT SURVEILLANCE PULSE AMPLIEIER DISCRIMINATOR (PAD) USED IN LEA & & REVISING \\
\hline $6-T F-554$ & 0 & 0 & FILTER MEDIA AND GAS BOTTLE REPLACEMENT PROCEDURE BELHAVEN MODEL BAT-FGM-4 & & DEVELOPING \\
\hline $6-\mathrm{TF}-555$ & 0 & 0 & CENTERLINE BUTTERFLY VALVE INSPECTION AND LUBRICATION & & TRANSFERED \\
\hline $6-\mathrm{VT}-159$ & A & 0 & HEGA FILTER IN-PLACE LEAK TEST & $10 / 26 / 97$ & ACTIVE \\
\hline $6-\mathrm{VT}-159 \mathrm{TA}$ & A & 2 . & APPENDIX TA, TANK EXHAUSTER HEGA LEAK TEST DATA SHEETS & $3 / 17 / 98$ & ACTIVE \\
\hline $6-\mathrm{VT}-162$ & B & 0 & ORGANIC VAPOR SAMPLING FOR TANK FARM EXHAUST STACKS & $6 / 05 / 98$ & ACTIVE \\
\hline $6-\mathrm{VT}-162 \mathrm{~A}$ & B & 0 & 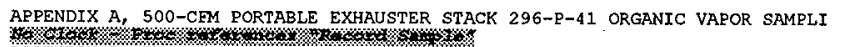 & $6 / 05 / 98$ & ACTIVE \\
\hline $6-\mathrm{VT}-162 \mathrm{~B}$ & B & 1 & APPENDIX B, EXHAUST STACK $296-A-42$ ORGANIC VAPOR SAMPLING DATA SHEETS & $6 / 05 / 98$ & ACTIVE \\
\hline $6-\mathrm{VT}-162 \mathrm{C}$ & A & 0 & APPENDIX C EXHAUST STACK 296-C-06 ORGANIC VAPOR SAMPLING DATA SHEETS & & DEVELOPING \\
\hline $6-V T-193$ & $\mathrm{c}$ & 1 & 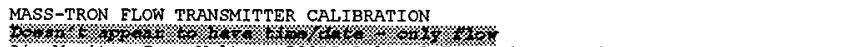 & $2 / 09 / 98$ & ACTIVE \\
\hline $6-V T-199$ & $\mathrm{~B}$ & 0 & 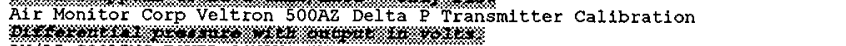 & $10 / 31 / 97$ & ACTIVE \\
\hline $6-V T-245$ & B & 0 & AY/AZ COOLING TOWER CONDUCTIVITY CONTROLLER SERVICING AND CALIBRATION & $10 / 31 / 97$ & ACTIVE \\
\hline \% & rom & & 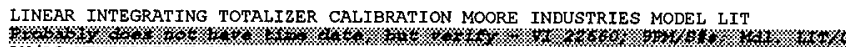 & $5 / 28 / 98$ & ACTIVE \\
\hline 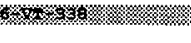 & $2 \%$ & 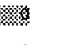 & $\begin{array}{l}\text { AY /AZ VENTILATION AND COOLING RAW WATER BADGER METER AND TRANSMITTER REVEN } \\
\text { S }\end{array}$ & $10 / 31 / 97$ & ACTIVE \\
\hline $6-V T-452$ & 0 & 0 & $\begin{array}{l}\text { CALIBRATE 4 - } 20 \text { MILIAMP RC SYSTEMS SIGNAL ISOLATOR OR AIR MONITOR CORPOR } \\
\text { H }\end{array}$ & $10 / 31 / 97$ & ACTIVE \\
\hline $6-V T-454$ & A & 0 & 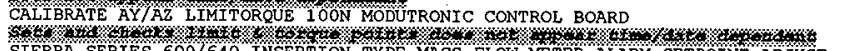 & $10 / 31 / 97$ & ACTIVE \\
\hline $6-V T P-128$ & A & 0 & SIERRA SERIES $600 / 640$ INSERTION TYPE MASS FLOW METER ALARM SETPOINT ADJUST & & DEVELOPING \\
\hline 7 -ABS-264 & A & 0 & ASBESTOS REMOVAL UTILIZING A GLOVE BAG & & DEVELOPING \\
\hline $7-A B S-265$ & A & 0 & ENCAPSULATION OF EXPOSED ASBESTOS (NO ASBESTOS REMOVED) FOR THE HANEORD SI & & DEVELOPING \\
\hline 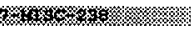 & (x) & 㗂 & 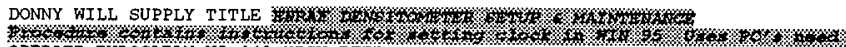 & $7 / 02 / 98$ & ACTIVE \\
\hline $7-M I S C-268$ & A & 0 & OFERATE EUROCLEAN UZ 948 HIGH EFEICIENCY PARTICULATE AIR FILTERED VACUUM & & DEVELOPING \\
\hline ARP-T-059-00001 & A & 2 & RESPOND TO ALARMS FOR THE CROSS SITE TRANSFER LINE SYSTEM & $3 / 30 / 98$ & ACTIVE \\
\hline
\end{tabular}

HNF-3410, Rev. 0 


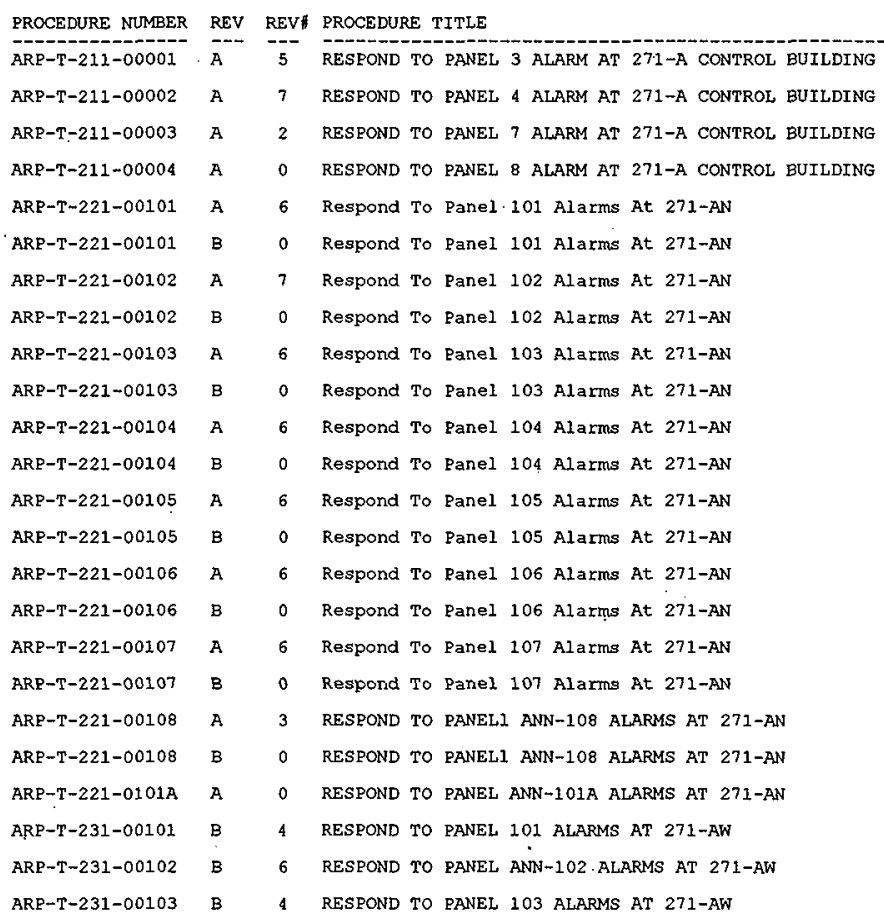

\begin{tabular}{|c|c|}
\hline RELEASE DATE & STATUS \\
\hline $5 / 28 / 98$ & ACTIVE \\
\hline $5 / 28 / 98$ & ACTIVE \\
\hline $9 / 29 / 97$ & ACTIVE \\
\hline $4 / 23 / 98$ & ACTIVE \\
\hline $4 / 24 / 98$ & ACTIVE \\
\hline & REVISING \\
\hline $4 / 27 / 98$ & ACTIVE \\
\hline & REVISING \\
\hline $4 / 27 / 98$ & ACTIVE \\
\hline & REVISING \\
\hline $4 / 27 / 98$ & ACTIVE \\
\hline & REVISING \\
\hline $4 / 27 / 98$ & ACTIVE \\
\hline & REVISING \\
\hline $4 / 24 / 98$ & ACTIVE \\
\hline & REVISING \\
\hline $4 / 27 / 98$ & ACTIVE \\
\hline & REVISING \\
\hline $3 / 04 / 98$ & ACTIVE \\
\hline & REVISING \\
\hline & DEVELOR ING \\
\hline $4 / 28 / 98$ & ACTIVE \\
\hline $6 / 25 / 98$ & ACTIVE \\
\hline $4 / 28 / 98$ & ACTIVE \\
\hline
\end{tabular}

HNF-3410, Rev, 0 
REV\# PROCEDURE TITLE

5 RESPOND TO PANEL 104 ALARMS AT 271-AW

\section{RESPOND TO PANEL 104 ALARMS AT 271 -AW}

105 ALARMS AT 271-AW

4 RESPOND TO PANEL 106 ALARMS AT 271-AW

4 RESPOND TO PANEL AUX ALARMS AT 271-AW

5 RESPOND TO MCS GRAPHIC \#03 LEAK DETECT ALARMS

4 RESPOND TO MONITOR CONTROL SYSTEM GRAEHIC \#05 RAW WATER SUPPLY ALARMS

RESPOND TO MONITOR CONTROL SYSTEM GRAPHIC \#06 EVAP AY1 ALARMS

RESPOND TO MONITOR CONTROL SYSTEM GRAEHIC \#07 EVAP AY2 ALARMS

RESPOND TO MONITOR CONTROL SYSTEM GRAPHIC \#O8 EVAP AZ1 ALARMS

RESPOND TO MONITOR CONTROL SYSTEM GRAPHIC 09 EVAP A22 ALARMS

RESPOND TO MONITOR CONTROL SYSTEM GRAPHIC \#10 RECIRC AY1 ALARMS

RESPOND TO MONITOR CONTROL SYSTEM GRAPHIC 11 RECIRC AY2 ALARMS

RESPOND TO MONITOR CONTROL SYSTEM GRAPHIC \#12 RECIRC AZ1 ALARMS

RESPOND TO MONITOR CONTROL SYSTEM GRAPHIC \#13 RECIRC AZ2 RLARMS

RESPOND TO MONITOR CONTROL SYSTEM GRAPHIC \#14 CHILLER ALARMS

RESPOND TO MONITOR CONTROL SYSTEM GRAPHIC \#15 PRIMARY VENT ALARMS

RESPOND TO MONITOR CONTROL SYSTEM GRAPHIC \#16 PRIMARY COOLING ALARMS

RESPOND TO MONITOR CONTROL SYSTEM GRAPHIC \#17 PRIMARY STACK ALARMS

RESPOND TO MONITOR CONTROL SYSTEM GRAPHIC \#18 PRIMARY VENT STACK ALARMS

RESPOND TO MONITOR CONTROL SYSTEM GRAPHIC \#19 BUILDING SUPPLY ALARMS

RESPOND TO MONITOR CONTROL SYSTEM GRAPHIC 20 BUILDING EXHAUST ALARMS

RESPOND TO MONITOR CONTROL SYSTEM GRAPHIC \#21 BUILDING STACK ALARMS

RESPOND TO MONITOR CONTROL SYSTEM GRAPHIC \#22 BUILDING VENT CONTROL ALARMS

RESPOND TO MONITOR CONTROL SYSTEM GRAPHIC \#23 DIESEL GENERATOR ALARMS
RELEASE DATE STATUS

4/28/98 ACTIVE

$4 / 29 / 98$

ACTIVE

$4 / 28 / 98$

ACTIVE

$3 / 03 / 98$

ACTIVE

$3 / 16 / 98$

ACTIVE

$3 / 10 / 98$

ACTIVE

$6 / 09 / 98$

ACTIVE

$6 / 09 / 98$

ACTIVE

$6 / 09 / 98$

ACTIVE

$6 / 09 / 98$

ACTIVE

$12 / 07 / 97$

ACTIVE

$12 / 07 / 97$

ACTIVE

$12 / 07 / 97$

ACTIVE

$12 / 07 / 97$

ACTIVE

$2 / 25 / 98$

ACTIVE

$6 / 04 / 98$

ACTIVE

$2 / 26 / 98$

ACTIVE

$3 / 17 / 98$

ACTIVE

7/02/98 ACTIVE

$3 / 09 / 98$ ACTIVE

12/07/97 ACTIVE

12/08/97 ACTIVE

$5 / 08 / 98$

ACTIVE

ACTIVE

HNF-3470, Rev. 0 


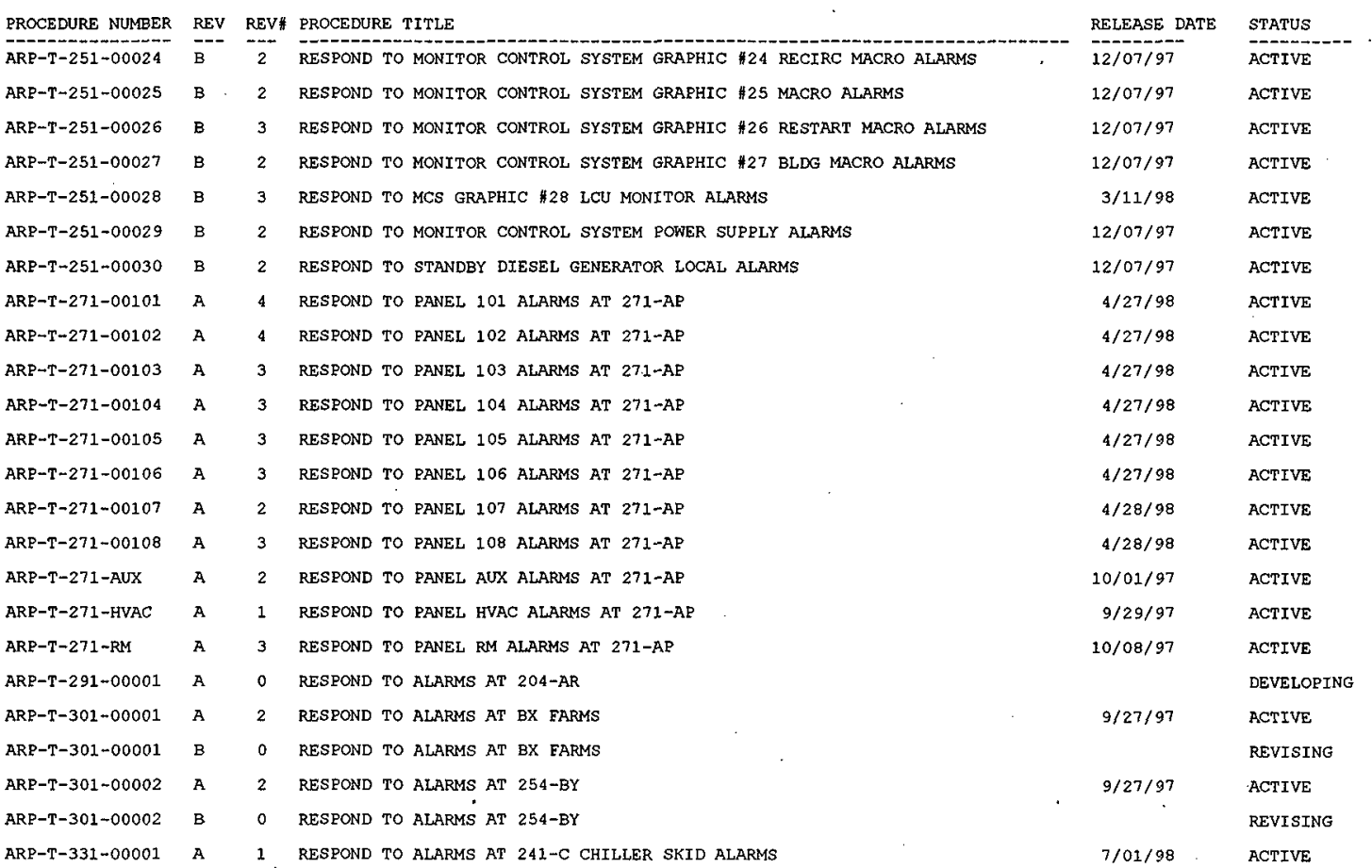

$H N F-3410$, Rev. 0 


\begin{tabular}{|c|c|c|c|c|c|}
\hline PROCEDURE NUMBER & REV & REV\# & PROCEDURE TITLE & RELEASE DATE & STATUS \\
\hline$A R E-T-331-00002$ & A & 2 & RESPOND TO ALARMS AT 241-C ELECTRICAL SKID & $7 / 27 / 98$ & ACTIVE \\
\hline ARP-T-331-00003 & A & 3 & RESPOND TO ALARMS AT MO- 211 WRSS CONTROL ROOM & $7 / 17 / 98$ & ACTIVE \\
\hline ARP-T-331-00004 & A & 0 & RESPOND TO ALARMS AT SERVICE BUILDING $241-\mathrm{C}-73$ & $5 / 05 / 98$ & ACT IVE \\
\hline ARP-T-331-00005 & A & 1 & RESPOND TO ALARMS AT ELECTRICAL EQUIPMENT SKID 241-AY-51 & $7 / 01 / 98$ & ACT IVE \\
\hline ARP-T-331-00006 & A & 3 & RESPOND TO ALARMS AT PROCESS CONTROL BUILDING 241-C-91 & $7 / 21 / 98$ & ACT IVE \\
\hline ARP-T-331-00007 & A & 2 & RESPOND TO WRSS DATA ACQUISITION SYSTEM COMPUTER ALARMS & $7 / 27 / 98$ & ACTIVE \\
\hline ARP-T-361-00001 & C & 0 & RESPOND TO ALARMS AT 271-CR & $9 / 22 / 97$ & ACTIVE \\
\hline ARP-T $-431-00001$ & A & 0 & RESPOND TO LOW NITROGEN PRESSURE ALARM SY-FARM & $6 / 25 / 97$ & ACTIVE \\
\hline ARE $-T-432-001$ & B & 0 & H2 CONCENTRATION HIGH & $6 / 11 / 97$ & ACT IVE \\
\hline ARP-T-432-003 & B & 1 & TANK DOME PRESSURE HIGH & $2 / 26 / 98$ & ACTIVE \\
\hline$A R P-T-432-004$ & B & 0 & VENT HEADER FLOW HIGH & $5 / 30 / 97$ & ACTIVE \\
\hline ARP $-T-432-005$ & B & 3 & MIT COLUMN STRAIN HIGH & $7 / 18 / 97$ & ACTIVE \\
\hline ARP-T-432-006 & B & 0 & VDTT COLUMN STRAIN HIGH & $5 / 30 / 97$ & ACTIVE \\
\hline ARP-T-432-007 & $\mathbf{B}$ & 0 & PUMP COLUMN STRAIN HIGH & $5 / 30 / 97$ & ACTIVE \\
\hline ARP $-T-432-010$ & B & 0 & WASTE TEMP HIGH & $5 / 30 / 97$ & ACTIVE \\
\hline ARP-T-432-011 & B & 0 & VENT HEADER FLOW LOW & $6 / 02 / 97$ & ACTIVE \\
\hline ARP-T-432-012 & $\mathrm{B}$ & 0 & PUMP MOTOR SPEED HIGH & $6 / 02 / 97$ & ACTIVE \\
\hline ARP-T $-432-014$ & $\mathbf{B}$ & 0 & PUMP MOTOR CURRENT HIGH & $8 / 28 / 97$ & ACT IVE \\
\hline ARP-T-432-015 & B & 0 & PUMP MOTOR OIL TEMPERATURE HIGH & $7 / 23 / 97$ & ACTIVE \\
\hline ARP-T-432-016 & $\mathbf{B}$ & 0 & PUMP COLUMN GAS PRESSURE LOW & $6 / 02 / 97$ & ACTIVE \\
\hline ARP-T-432-017 & B & 0 & MOISTURE IN PUMP MOTOR OII HIGH & $6 / 02 / 97$ & ACTIVE \\
\hline ARP-T-432-019 & B & 0 & DUAL PLC FAILURE & $6 / 11 / 97$ & ACTIVE \\
\hline ARP-T-432-020 & B & 0 & STATION 5 COMPUTER LOCKUP OR CRASH & $3 / 18 / 97$ & ACTIVE \\
\hline ARP-T-432-021 & B & 0 & STATION 8 COMPUTER LOCKUP OR CRASH & $6 / 02 / 97$ & ACTIVE \\
\hline
\end{tabular}

HNF -3410 , Rev. 0 
ARP-T-432-026

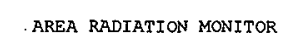

$\begin{array}{ll}11 / 06 / 96 & \text { REVISING } \\ & \text { ACTIVE } \\ 4 / 15 / 98 & \text { REVISING } \\ 6 / 22 / 98 & \text { ACTIVE } \\ 7 / 19 / 95 & \text { ACTIVE } \\ 7 / 19 / 95 & \text { REVISING } \\ & \text { ACTIVE } \\ 1 / 08 / 98 & \text { REVISING } \\ & \text { REVISING } \\ 9 / 27 / 97 & \text { RCTIVE } \\ & \text { REVISING } \\ 2 / 27 / 98 & \text { REVISING } \\ & \text { ACTIVE } \\ 2 / 06 / 98 & \text { REVISING } \\ & \text { ACTIVE } \\ & \text { REVISING } \\ & \text { ACTIVE } \\ & \text { REVISING }\end{array}$

HNF-3410, Rev. 0 


\begin{tabular}{|c|c|c|c|c|c|}
\hline PROCEDURE NUMBER & REV & REV\# & PROCEDURE TITLE & RELEASE DATE & STATUS \\
\hline ATP-040-001 & & 2 & STANDARD-E HYDROGEN MONITORING SYSTEM FIELD ACCEPTANCE TEST PROCEDURE & $8 / 05 / 97$ & ACTIVE \\
\hline ATP-040-002 & A & 5 & SALTWELL JET PUMP ASSEMBLY RUN-IN ACCEPTANCE TEST PROCEDURE & $4 / 07 / 98$ & ACTIVE \\
\hline CPS-T-149-00010 & $H$ & 0 & WASTE STORAGE IN DOUBLE-SHELL TANKS AND ASSOCIATED EQUIEMENT & $8 / 25 / 97$ & ACTIVE \\
\hline OSD-T-151-00007 & $H$ & 20 & OPERATING SPECIFICATIONS FOR $241-A N, A P, A W, A Y, A 2 \& S Y$ TANK FARMS & $6 / 24 / 98$ & ACTIVE \\
\hline OSD-T-151-00008 & $\mathbf{E}$ & 2 & FOR THE 204-AR WASTE FOR 204-AR WASTE UNLOADING FACILITY & $10 / 20 / 94$ & ACTIVE \\
\hline OSD-T-151-00010 & D & 6 & OPERATING SPECIFICATIONS FOR PRESSURE CHECKING OF ALL DIRECT BURIED AND CR & $6 / 23 / 98$ & ACTIVE \\
\hline OSD-T-151-00011 & $\mathrm{C}$ & 4 & OPERATING SPECIFICATION FOR SALTWELL RECEIVER VESSELS & $7 / 22 / 96$ & ACTIVE \\
\hline OSD-T-151-00012 & D & 2 & OPERATING SPECIFICATIONS FOR THE 242-A EVAPORATOR & $1 / 22 / 96$ & ACTIVE \\
\hline OSD $-T-151-00013$ & D & 25 & OPERATING SPECIFICATION FOR SINGLE-SHELL WASTE STORAGE TANKS & $10 / 02 / 97$ & ACTIVE \\
\hline OSD $-\mathrm{T}-151-00014$ & A & 5 & OPERATING SPECIFICATION FOR THE 244-AR VAULT FACILITY & $12 / 21 / 94$ & ACTIVE \\
\hline $0 S \mathrm{D}-\mathrm{T}-151-00015$ & B & 9 & OPERATING SPECIFICATIONS FOR MISCELLANEOUS FACILITIES & $1 / 11 / 96$. & ACTIVE \\
\hline OSD-T-151-00016 & $\mathrm{C}$ & 2 & ORERATING SPECIEICATIONS EOR THE 241-A-702 VESSEL VENTILATION SYSTEM & $20 / 20 / 94$ & ACTIVE \\
\hline OSD -T-151-00017 & $\mathbf{D}$ & 10 & OPERATING SPECIFICATIONS FOR AGING-WASTE OPERATIONS IN 241-AY AND 241-AZ & $10 / 01 / 97$ & ACTIVE \\
\hline OSD-T-151-00018 & $\mathbf{F}$ & 0 & OPERATING SPECIFICAIONS FOR ACTIVE POND AND CRIB DISPOSAL SITE & $7 / 05 / 95$ & ACTIVE \\
\hline OSD-T-151-00019 & $\mathbf{B}$ & $i$ & OPERATING SPECIFICAIONS FOR $W-030$ & $1 / 06 / 98$ & ACTIVE \\
\hline OSD-T-151-00025 & A & 0 & OPERATING SPECIFICATIONS FOR THE BURIRL GROUNDS & & DEVELOPING \\
\hline OSD-T-151-00026 & A & 0 & OPERATING SPECIFICATIONS FOR THE TRANSURANIC STORAGE AND ASSAY FACILITIES & & DEVELORING \\
\hline OSD-T-151-00027 & A. & 0 & OPERATING SPECIFICATIONS FOR THE 213-W RADIOACTIVE SOLID WASTE COMPACTOR $F$ & & DEVELOPING \\
\hline OSD-T-151-00029 & B & 1 & OPERATING SFECIFICATIONS FOR THE LIQUID EEFLUENT RETENTION FACILITX & $5 / 02 / 97$ & ACTIVE \\
\hline OSD-T-151-00030 & $\mathrm{B}$ & 27 & OPERATING SPECIFICATIONS FOR WATCH LIST TANKS & $6 / 01 / 98$ & ACTIVE \\
\hline OSD-T-151-00031 & B & 4 & OPERATING SPEEICIATIONS FOR TANK FARM LEAK DETECTION & $12 / 04 / 96$ & ACTIVE \\
\hline OTP-020-001 & A & 0 & PEREORM OPERATIONAL TESTING FOR THE LR-56 CASK TRAILER & & DEVELOPING \\
\hline OTP-060-001 & & 2 & OPERATION TEST PROCEDURE FOR THE 241-A-101 500 CFM EXHAUSTER & $10 / 18 / 96$ & ACTIVE \\
\hline OTP-060-002 & A & 3 & PROCESS TEST TANK $241-C-106 \quad 296-\mathrm{P} 16$ EXHAUSTER OUTAGE & $6 / 06 / 97$ & ACTIVE \\
\hline
\end{tabular}

HNF-34T0, Rev. 0 
PROCEDURE NUMBER REV REV\# PROCEDURE TITLE

OTP-200-001 A 14

OTP-200-002

OTP-200-003

OTP-200-004

OTP-210-001

OTP-220-001

OTP-320-001

OTP-320-002

OTP-320-003

OTP-320-004

OTP-320-005

OTP-320-006

OTP-320-007

OTP-320-008

OTP-320-009

OTP-320-010

OTP-320-011

OTP-W030-001

OTP-W030-003

POTP-T-W030-008

\%

PSCP-3-051

2 B

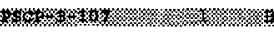

10.0\%
OPERATION TEST PROCEDURES FOR THE A-101 PUMPING AND INSTRUMENTATION CONTRO

OPERATIONAL TEST PROCEDURE FOR THE BELHAVEN FLAMMABLE GAS MONITOR MODEL FG

OPERATIONAL TEST PROCEDURE FLAMMABLE GAS MONITOR MODEL

OPERATION TEST PROCEDURE FOR 600 SERIES PUMPING AND INSTRUMENTATION CONTRO

AZ-101 MIXER PUMP OPERATIONAL TEST PROCEDURE

DG-A-002, BACKUP DIESEL GENERATOR SET

W-320 ANNULUS VENTILATION SYSTEM OPERATIONAL TEST

W-320 SLURRY TRANSFER SYSTEM PREOPERATIONAI TEST

W-320 SUPERNATE TRANSFER SYSTEM PREOPERATIONAL TEST

W-320, TANK 241-C-106 VENTILATION EXHAUST SYSTEM RREOPERATIONAL TEST

TANK 241-C-106 IN-TANK IMAGING SYSTEM OPERATIONAL TEST PROCEDURE

PROJECT $\omega-320$ CATHODIC RROTECTION SYSTEM OPERATIONAL TEST PROCEDURE

OPERATIONAL TEST EMERGENCY BACKUP 241-C-106 1000 CEM EXHAUSTER

ORERATIONAL TEST EMERGENCY BACKUP 241-AY-102 1000 CEM EXHAUSTER

OPERATIONAL TEST EMERGENCY BACKUP 241-AY-102 ANNULUS 1000 CEM EXYAAUSTER

W-320 ENRAF SERIES 854 DENSITOMETER OPERATIONAL TEST

W-320 WRSS 296-C-106 HVAC SYSTEM (TIE-IN) OPERATIONAL TEST

W-030 OPERATIONAL TEST PROCEDURE INTEGRATED SYSTEM TEST

CATHODIC PROTECTION SYSTEMS OPERATIONAL TEST PROCEDURE

W-030 PRE-OPERATIONAL TEST PROCEDURE INTEGRATED SYSTEM TEST

FAIRCHI LD MODELS T-5100B-1, T-5100B-4,5200, AND T-5600 I/P TRANSDUCERS HANFORD-BUILT SURVEILLANCE PULSE AMPLIFIER DISCRIMINATOR (PAD) USED IN LEA

SAIC/RADECO MODEL H-809B, H809C, H810, 787, H-809V, HD-29, HD-29A, HD-66A. tw.

MM LEEDS AND NORTHRUP SPEEDOMAX M MARK III RECORDER

Hook at
RELEASE DATE

$12 / 23 / 97$

$11 / 22 / 96$

$2 / 28 / 97$

$4 / 27 / 98$

$1 / 21 / 98$

$5 / 27 / 97$

$2 / 12 / 98$

$7 / 18 / 98$

$6 / 17 / 98$

$7 / 15 / 98$

$5 / 11 / 98$

$5 / 19 / 98$

$7 / 02 / 98$

$7 / 02 / 98$

$7 / 02 / 98$

$6 / 24 / 98$

$7 / 22 / 98$

$7 / 29 / 97$

$2 / 19 / 98$

$3 / 06 / 95$

$10 / 20 / 95$

$5 / 27 / 97$
STATUS

ACTIVE

ACTIVE

ACTIVE

ACTIVE

ACTIVE

ACTIVE

ACTIVE

ACTIVE

ACTIVE

ACTIVE

ACTIVE

ACTIVE

ACTIVE

ACTIVE

ACTIVE

ACTIVE

DEVELOPING

ACTIVE

DEVELOPING

ACTIVE

ACTIVE

ACTIVE

ACTIVE

ACTIVE

HNF-3410, Rev. 0 


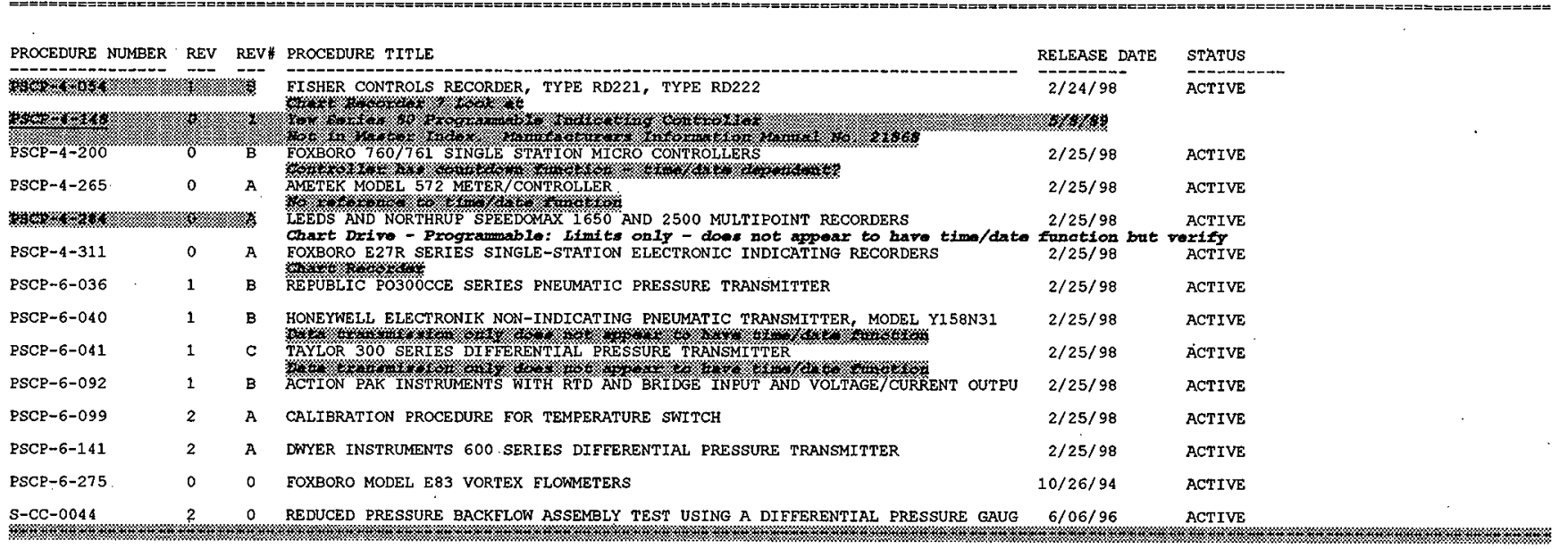

HNF -3410, Rev. 0 


\section{DISTRIBUTION SHEET}

\begin{tabular}{|c|c|c|c|c|c|}
\hline \multirow[b]{2}{*}{ Distribution } & \multirow{2}{*}{\multicolumn{3}{|c|}{$\begin{array}{l}\text { From } \\
\text { Data Management/Technical } \\
\text { Basis and Planning }\end{array}$}} & \multicolumn{2}{|l|}{ Page 1 of 1} \\
\hline & & & & \multicolumn{2}{|c|}{ Date $\quad 09 / 21 / 98$} \\
\hline \multicolumn{4}{|c|}{ Project Title/Work Order } & \multicolumn{2}{|c|}{ EDT No. $\quad$ EDT-622476 } \\
\hline $\begin{array}{l}\text { HNF-3410, Rev. 0, "Year } 2000 \\
\text { Report" }\end{array}$ & Maintenance & rocedure & Review & \multicolumn{2}{|c|}{ ECN No. $\quad$ N/A } \\
\hline Name & MSIN & $\begin{array}{l}\text { Text } \\
\text { With } \\
\text { All } \\
\text { Attach. }\end{array}$ & Text Only & $\begin{array}{c}\text { Attach./ } \\
\text { Appendix } \\
\text { Only }\end{array}$ & $\begin{array}{l}\mathrm{EDT} / \mathrm{ECN} \\
\text { Only }\end{array}$ \\
\hline
\end{tabular}

Lockheed Martin Hanford, Corp.

M. R. Adams

R. B. Bass

J. W. Hunt

E. I. Husa

J. A. Johnston

D. B. Smet

J. K. Wylie

T.C.S.R.C.

$\begin{array}{ll}\text { R2 }-12 & X \\ \text { H7 }-06 & X \\ \text { R2-12 } & X \\ \text { R2-11 } & X \\ \text { R2-12 } & X \\ \text { R1 }-56 & X \\ \text { H7 }-06 & X \\ \text { R1-10 } & X\end{array}$

Lockheed Martin Services, Inc. Central Files

B1-07 $X$

